\title{
The rotational profiles of cluster galaxies
}

\author{
Lawrence E. Bilton ${ }^{\circledR},{ }^{\star}$ Matthew Hunt, Kevin A. Pimbblet and Elke Roediger \\ E.A. Milne Centre for Astrophysics, The University of Hull, Cottingham Road, Kingston upon Hull HU6 7RX, UK
}

Accepted 2019 October 2. Received 2019 October 2; in original form 2019 August 20

\begin{abstract}
We compile two samples of cluster galaxies with complimentary hydrodynamic and $\mathrm{N}$-body analysis using FLASH code to ascertain how their differing populations drive their rotational profiles and to better understand their dynamical histories. We select our main cluster sample from the X-ray Galaxy Clusters Database (BAX), which are populated with Sloan Digital Sky Survey (SDSS) galaxies. The BAX clusters are tested for the presence of substructures, acting as proxies for core mergers, culminating in sub-samples of eight merging and 25 non-merging galaxy clusters. An additional sample of 12 galaxy clusters with known dumbbell components is procured using galaxy data from the NASA/IPAC Extragalactic Database (NED) to compare against more extreme environments. BAX clusters of each sample are stacked on to a common RA-Dec. space to produce rotational profiles within the range of 0.0-2.5 $r_{200}$. Merging stacks possess stronger core rotation at $\lesssim 0.5 r_{200}$ primarily contributed by a red galaxy subpopulation from relaxing core mergers; this is alongside high rotational velocities from blue galaxy sub-populations, until they mix and homogenize with the red sub-populations at $\sim r_{200}$, indicative of an infalling blue galaxy sub-population with interactive mixing between both sub-populations at $\gtrsim r_{200}$. FLASH code is utilized to simulate the merger phase between two originally independent clusters and test the evolution of their rotational profiles. Comparisons with the dumbbell clusters leads to the inference that the peculiar core rotations of some dumbbell clusters are the result of the linear motions of core galaxies relaxing on to the potential during post second infall.
\end{abstract}

Key words: galaxies: clusters: general-galaxies: elliptical and lenticular, cD-galaxies: kinematics and dynamics.

\section{INTRODUCTION}

Galaxy clusters are large and dense realms in space which anistropically coalesce along the convergence of independent filaments through hierarchical merger events, resulting in the induction of random motions in their member galaxies (e.g. Bond, Kofman \& Pogosyan 1996; Springel et al. 2005; Kravtsov \& Borgani 2012). These large collections of matter are home to strong gravitational potentials that cause the further perturbation of galaxies from the Hubble flow (Regos \& Geller 1989). As a result, galaxy clusters seemingly play host to environmental effects that are pivotal in the understanding of the evolution of galaxies through an assumption of fixed stellar mass: the transition from late-type to early-type galaxies towards the cluster's centre with the morphology-density relation (e.g. Oemler 1974; Dressler 1980; Postman \& Geller 1984); the observed bimodality of the colour-density relation (Hogg et al. 2003, 2004); the consistent decrease in the fraction of star-forming galaxies in cluster cores (e.g. Lewis et al. 2002; Gómez et al. 2003;

^E-mail: 1.bilton@2016.hull.ac.uk
Bamford et al. 2009; von der Linden et al. 2010); a galaxy infalling on to a cluster potential experiencing ram-pressure stripping due to interacting with the intracluster medium (ICM) (e.g. Gunn \& Gott 1972; Poggianti et al. 2017; Sheen et al. 2017).

The hierarchical nature of galaxy cluster formation lends itself to the existence of physical substructures (Geller \& Beers 1982; Dressler \& Shectman 1988). Therefore, the aforementioned environmental effects on galaxy evolution can be scaled down to the smaller substructure environments within a cluster. We can use the presence and strength of this substructuring within the cluster to delineate differing environments (i.e. merging or nonmerging). The substructures that reside at larger radii from the cluster centre are smaller galaxy groups that cause 'pre-processing' (Berrier et al. 2009; Bahé et al. 2013); smaller scale premature evolution of galaxies due to localized galaxy-galaxy interactions (see Moore et al. 1999). Pre-processing is considered to be a common occurrence in order to account for the swift changes in star formation and colour fractions as galaxies transition from the field (e.g. see Haines et al. 2015; Bilton \& Pimbblet 2018).

Perhaps one of the striking features of many galaxy clusters is the presence of overtly bright giant early-type galaxies, commonly 
with an extended diffuse region, that lie within the dynamical centres of their host cluster (Quintana \& Lawrie 1982), otherwise known as the brightest cluster galaxy (BCG). The formation mechanism for BCGs has been key point of contention. One such model is galactic cannibalism (Ostriker \& Tremaine 1975), whereby galaxies infall and accumulate at the bottom of the potential well through dynamical friction. An alternatively favoured model is rapid hierarchical galaxy-galaxy merging into an ensemble of subgroupings of galaxies of similar size prior to collapse on to the bottom of the potential (Merritt 1985). Testing of these models has often yielded mixed results; galactic cannibalism is deemed too slow in order to build a BCG within a reasonable time-scale with the observed luminosities (e.g. Lauer 1988; Dubinski 1998); hierarchical galaxy merging events alone do not assemble enough subgroupings with calculations to our current epoch (Collins et al. 2009). However, despite these shortfalls, there is evidence for clusters to have had merger events over their histories with the observations of BCGs with multiple cores (e.g. Oegerle \& Hill 1992; Laine et al. 2003). There is also convincing evidence of core-core pre-merger, ongoing merger, post-merger activity between two originally independent potential wells, with high peculiar velocities of BCGs, that indicate perturbations from their original geometric and kinematic centres (e.g. Quintana, Ramirez \& Way 1996; Smith et al. 2005; Pimbblet, Roseboom \& Doyle 2006; Shan et al. 2010; Lakhchaura et al. 2013; Caglar \& Hudaverdi 2017). These systems with multiple-core BCGs are sometimes known as 'Dumbbell Clusters'.

If we assume these dumbbell clusters arise from two originally independent sub-clusters interacting off-axially, then the strength and presence of their resultant momenta could leave an imprint on to their line-of-sight velocities, producing some sort of 'global cluster rotation' (Ricker 1998). Due to the apparent random motions of cluster galaxies, the idea of galaxy clusters supported by rotational energies was excused for a pressure-based model. However, once thought indistinguishable from the cluster galaxy kinematics, there have since been several works that have observed global rotation (e.g. Materne \& Hopp 1983; Oegerle \& Hill 1992; Hwang \& Lee 2007; Tovmassian 2015; Manolopoulou \& Plionis 2017). One could argue that the source of cluster rotation is from the Universe possessing its own angular momentum and donating it on to celestial bodies during their formation ( $\mathrm{Li}$ 1998; Godłowski et al. 2003; Godłowski, Szydłowski \& Flin 2005). However, to account for the strong peculiar velocities from relatively recent histories, galaxy cluster rotation could be derived from the merging processes between two clusters (Peebles 1969; Ricker 1998): offaxis tidal interactions from two independent deep potential wells. Observations of such events/relics would only be pragmatic by observing the ICM due to the high collisional probability of particles that produce X-rays, where the more sparse cluster galaxies are found to be collisionless on equal time-scales; global angular momentum observed via the galaxies is transient (White \& Fabian 1995; Roettiger, Loken \& Burns 1997; Roettiger \& Flores 2000). Indeed, recent simulation studies show how the ICM could be used to determine bulk cluster rotation dynamics (see Baldi et al. 2017, 2018). In addition, there is the very sensible notion that the accretion of mass through the filaments during cluster assembly is the primary driver of momentum donation to these systems (see Song et al. 2018). One immediate method that could be used to infer a cluster's global rotation is the use of a geometrical technique known as 'perspective rotation': peculiar motion measurements taken from the mean radial velocities to determine the transverse motion by artificially rotating the galaxy cluster on the plane of the sky (Feast, Thackeray \& Wesselink 1961).
Therefore, in this paper we aim to establish whether or not the global rotational dynamics of clusters correlate with their subpopulations and if the presence of a dumbbell BCG core imprints a cluster evolutionary mechanism on to the global rotational profile. This will be achieved by utilizing a 'perspective rotation' technique, which infers the presence of cluster rotation through the comparative radial velocity differences between two semicircles divided by the cluster centre (see Manolopoulou \& Plionis 2017; MP17 hereafter). We present a complementary suite of galaxy data from the Sloan Digital Sky Survey (SDSS) and NASA/IPAC Extragalactic Database (NED) to form a membership of clusters into two samples of those that do, and do not, host a dumbbell nucleus. An elaboration on how these data were acquired can be found in Section 2. An explanation of the methods used to output our global rotation profile analysis, along with our results, is outlined in Section 3. In addition to our array of observational data, we utilize $3 \mathrm{D}$ hydrodynamics and $\mathrm{N}$-body simulations to determine the impact of idealized binary cluster mergers on global rotational profiles. The comparison of our observational rotational analysis with our 3D hydrodynamic and $N$-body simulations are elaborated in Section 4. In Section 5, we conclude with a discussion and summary of our findings in this work.

Throughout this work we assume a flat Lambda cold dark matter model of cosmology with $\Omega_{\mathrm{M}}=0.3, \Omega_{\Lambda}=0.7, H_{0}=100 h \mathrm{~km} \mathrm{~s}^{-1}$ $\mathrm{Mpc}^{-1}$, where $h=0.7$.

\section{THE DATA SUITE}

We compile two samples of clusters to allow for a more comprehensive study of the effects of cluster rotation: a sample of bright $\mathrm{X}$-ray-selected clusters utilizing the X-ray Cluster Database (BAX; Sadat et al. 2004), a curated repository linking X-ray data from multiple instrumental sources; and a sample of dumbbell clusters catalogued by Gregorini et al. (1992, 1994). Each X-ray-selected cluster is then built from the BAX centre and defined by galaxies from SDSS Data Release 8 (DR8; Aihara et al. 2011) cross-matched with the MPA-JHU Value Added Catalogue (Kauffmann et al. 2003; Brinchmann et al. 2004; Tremonti et al. 2004). The dumbbell clusters are assembled via the procurement of NED galaxies that lie within 30 arcmin of the NED-defined centres of the 12 dumbbell clusters, similar to the work presented by Pimbblet (2008).

We initialize our cluster sample with the BAX data base by employing an X-ray luminosity range of $1<L_{\mathrm{X}} \leq 20 \times 10^{44} \mathrm{ergs}^{-1}$ that lie within the redshift range of $0.0<z \leq 0.15$. These limits ensure we are selecting the most massive clusters while garnering a significant number of clusters sampled across a variety of dynamical states in $z$-space from a finite epoch range of cluster evolution. Once parsed through BAX, the applied limits provide an initial sample size of 481 clusters. For each of the BAX clusters an initial radial limit of $\leq 10 \mathrm{Mpc} h^{-1}$ is applied to DR8 galaxies from the cluster centre, calculated with our outlined cosmology (Wright 2006). The cluster sample is iterated through to have their global mean recession velocities $\left(\overline{c z}_{\text {glob }}\right)$ and velocity dispersions $\left(\sigma_{\text {glob }}\right)$ calculated for galaxies $\leq 1.5 \mathrm{Mpc} h^{-1}$ from the cluster centre by computing the square root of the biweight mid-variance (Beers, Flynn \& Gebhardt 1990). We then proceed to define the cluster boundary in velocity space as a function of projected radius $R$ with surface caustics, in concordance with the methodology of Diaferio \& Geller (1997) and Diaferio (1999). Velocity limits of $\Delta V= \pm 1500 \mathrm{~km} \mathrm{~s}^{-1}$ are imposed upon each cluster in the sample as a conservative threshold to increase confidence against interlopers contaminating the DR8 
assembled clusters, with

$$
\Delta V=c\left(\frac{z_{\mathrm{gal}}-z_{\mathrm{clu}}}{1+z_{\mathrm{clu}}}\right) \text {. }
$$

However, it should be noted that this limit can potentially omit genuine members from those systems that are actively relaxing on to a cluster potential due to their greater dispersion of galaxies as an echo from two originally independent sub-clusters coalescing on to each other. The surface caustic profiles are then determined with the remaining galaxies for each cluster, allowing for estimations of $M_{200}$ and $r_{200}$, the cluster masses and radii for when the density is 200 times the critical density of the universe for our flat cosmology (Gifford \& Miller 2013; Gifford, Miller \& Kern 2013). Cluster candidates are ignored if their initial richness is $<50$ at $\leq r_{200}$, or if they are found within the Einasto et al. (2001) supercluster catalogue to possesses overlapping structures. The resultant cluster sample size provided by the BAX-DR8 galaxies is 33 , which is found to be mass complete at $\log _{10}\left(M_{*}\right) \geq 10.2$. The final compilation of BAXdefined clusters built from DR8 galaxies can be found in Table 1 .

The 12 dumbbell clusters are initialized with the NED galaxies that reside within 30 arcmin from the NED defined centres. The NED galaxies associated with each cluster are then run through the same process as the DR8 galaxies above. The NED-defined clusters built from the NED galaxies, along with their calculated values, can be found in Table 2 .

\section{CLUSTER ROTATION AND ENVIRONMENTAL EFFECTS}

It has already been noted in Section 1 how one might expect galaxy clusters to have gained their momentum. To study how different cluster activity states drive the dynamical side effects on to cluster galaxy sub-populations we utilize the BAX-selected clusters to compile two sub-samples, those that possess substructure against those without, as determined by the Dressler \& Shectman (1988) test ( $\Delta$-test hereafter) which is outlined in Section 3.1 below. The methodology outlined by MP17, elaborated in Section 3.2, is applied to construct the rotational curves determined through their artificial transverse rotation on the plane of the sky to study how the cluster galaxy sub-populations respond to global cluster rotation and level of substructuring. From this method, we determine the rotational profiles as a function of projected radius of the clusters from both our samples, as well as producing composite profiles for merging and non-merging (i.e. level of activity) sub-samples for the BAXselected clusters (see Sections 3.3 and 3.4), allowing us to compare the angular momentum of galaxy clusters between different possible states of merger activity and how cluster galaxy sub-populations drive the resultant profiles.

\subsection{The $\Delta$-test}

The use of a geometric perspective motion effect can lead to spurious detections of ambient cluster rotation in the presence of strong galaxy-galaxy merger activity from a merging cluster. However, within this work, we aim to establish the rotational velocities (see 3.2) as a function of radius to determine how the strength of the global cluster rotation varies between merging and nonmerging environments. Therefore, to test how cluster activity can alter the rotational dynamics of clusters and thereby affect the evolution of the cluster galaxies, we incorporate the $\Delta$-test for substructure on galaxies within $1.5 \mathrm{Mpc} h^{-1}$ (defined as $N_{\text {glob }}$ ) of the BAX- and NED-defined cluster centres in order to delineate between merging and non-merging environments. The $\Delta$-test is a commonly used and very robust tool for indicating substructure, with substructure detections reaching $>99$ per cent confidence when applied on cluster galaxy sample sizes of $N_{\text {glob }} \geq 60$ (e.g. Pinkney et al. 1996; Pimbblet 2008; Bilton \& Pimbblet 2018; Song et al. 2018). Therefore, we apply the $\Delta$-test on to each galaxy and their $N_{n n}=\sqrt{N_{\text {glob }}}$ nearest neighbours. The localized kinematics are determined and then compared against the global values,

$\delta_{i}^{2}=\left(\frac{N_{n n}+1}{\sigma_{\text {glob }}^{2}}\right)\left[\left(\overline{c z}_{\text {local }}-\overline{c z}_{\text {glob }}\right)^{2}+\left(\sigma_{\text {local }}-\sigma_{\text {glob }}\right)^{2}\right]$,

where $\delta$ measures the deviation in the small region around the galaxy compared to the global cluster values at $\leq 1.5 \mathrm{Mpc} h^{-1}$. The application of the $\Delta$-test to the BAX sample leads to a merging sub-sample size of eight clusters and a non-merging sub-sample size of 25 clusters, the resultant values for which can be found in Table 1. Example phase-space diagrams, along with their respective caustics, produced from each sub-sample of clusters are presented in Fig. 1 to illustrate the membership and spread of galaxies for each cluster and their local environments.

\subsection{The Manolopoulou and Plionis method}

In order to determine our averaged cluster galaxy rotational profiles, we employ the methodology of MP17, which utilizes the geometrical 'Perspective Rotation'. Assuming an ideal case where the rotational axis of a cluster is perpendicular to our line of sight (i.e. $\phi=0^{\circ}$ ), we split our cluster into two semicircles vertically down the X-ray-defined centre and determine their line-of-sight velocities of the member galaxies with respect to their angle $\mu$ from the origin. The mean velocity of each semicircle $\left(\left\langle v_{1}\right\rangle,\left\langle v_{2}\right\rangle\right)$ is then determined in equation (3), enabling observations in how the difference in the mean velocities of each semicircle $\left(v_{\text {diff }}=\left\langle v_{1}\right\rangle-\right.$ $\left.\left\langle v_{2}\right\rangle\right)$ vary as we project the average proper motions of the galaxies through the transverse rotation of galaxies in $\theta=10^{\circ}$ increments. Therefore, for each semicircle we apply iteratively

$\left\langle v_{1,2}\right\rangle=\frac{1}{N} \sum_{i=1}^{N} \Delta V_{i} \cos \left(90^{\circ}-\mu_{i}\right)$,

where $\Delta V_{i}$ is the line-of-sight velocity from equation (1) for the galaxy $z_{\mathrm{gal}, i}$, and the angle from the origin $\mu_{i}$ operates between $0^{\circ}$ and $180^{\circ}$ for each semicircle. This means that $v_{\text {diff }}$ can be determined for each angle $\theta$, leading to the uncertainties of each semicircle being propagated through for each angle $\theta$ as

$\sigma_{\theta}=\sqrt{\frac{\sigma_{v, 1}^{2}}{n_{1}}+\frac{\sigma_{v, 2}^{2}}{n_{2}}}$,

where $\sigma_{v}$ is the velocity dispersion and $n$ is the galaxy number for each semicircle 1 and 2 at each angle of $\theta$.

Finally, we assume the maximum $v_{\text {diff }}(\theta)$ provides the rotational velocity $v_{\text {rot }}=\operatorname{MAX}\left[v_{\text {diff }}(\theta)\right]$, which consequently provides the angle of the rotational axis in the plane of the sky $\theta_{\text {rot }}$. Therefore, for our global cluster definition, we determine rotational values for each cluster from our BAX and NED samples which are computed using equation (3) with galaxies that lie $\leq 1.5 \mathrm{Mpc}^{-1}$ from their respective cluster centres, thus providing the final cumulative global cluster rotational velocities and angle of the rotational axes for each, which are denoted as $v_{\text {glob }}$ and $\theta_{\text {glob }}$, respectively. The statistical significance of the presence of rotation from our global definition is calculated for galaxies from both our BAX and NED cluster 
Table 1. The mass-complete BAX cluster sample. The J2000 coordinates and X-ray luminosity values are taken from BAX. The methodology for the determination of kinematic and global rotational values can be found in Sections 2 and 3.2. $\chi_{\text {id }}^{2} / \chi_{\text {rd }}^{2}$ is the ratio of the $\chi^{2}$ statistic between ideal and random rotation curves with $P(\mathrm{KS})$ being the two-sample Kolmogorov-Smirnov test of significance in rotation. MP17 defines a strict criterion as $P(\mathrm{KS})<0.01$ and $\chi_{\mathrm{id}}^{2} / \chi_{\mathrm{rd}}^{2} \leq 0.2$, alongside a loose criterion as $P(\mathrm{KS})<0.01$ and $\chi_{\mathrm{id}}^{2} / \chi_{\mathrm{rd}}^{2} \leq 0.4$, for determining the presence of cluster rotation. The $P(\Delta)$ values represent the significance of substructuring with respect to the $\Delta$-test in equation (2). $P(\Delta) \ll 0.01$ and $P(\mathrm{KS}) \ll 0.01$ is strongly indicative of substructure and rotation with values smaller three d.p.

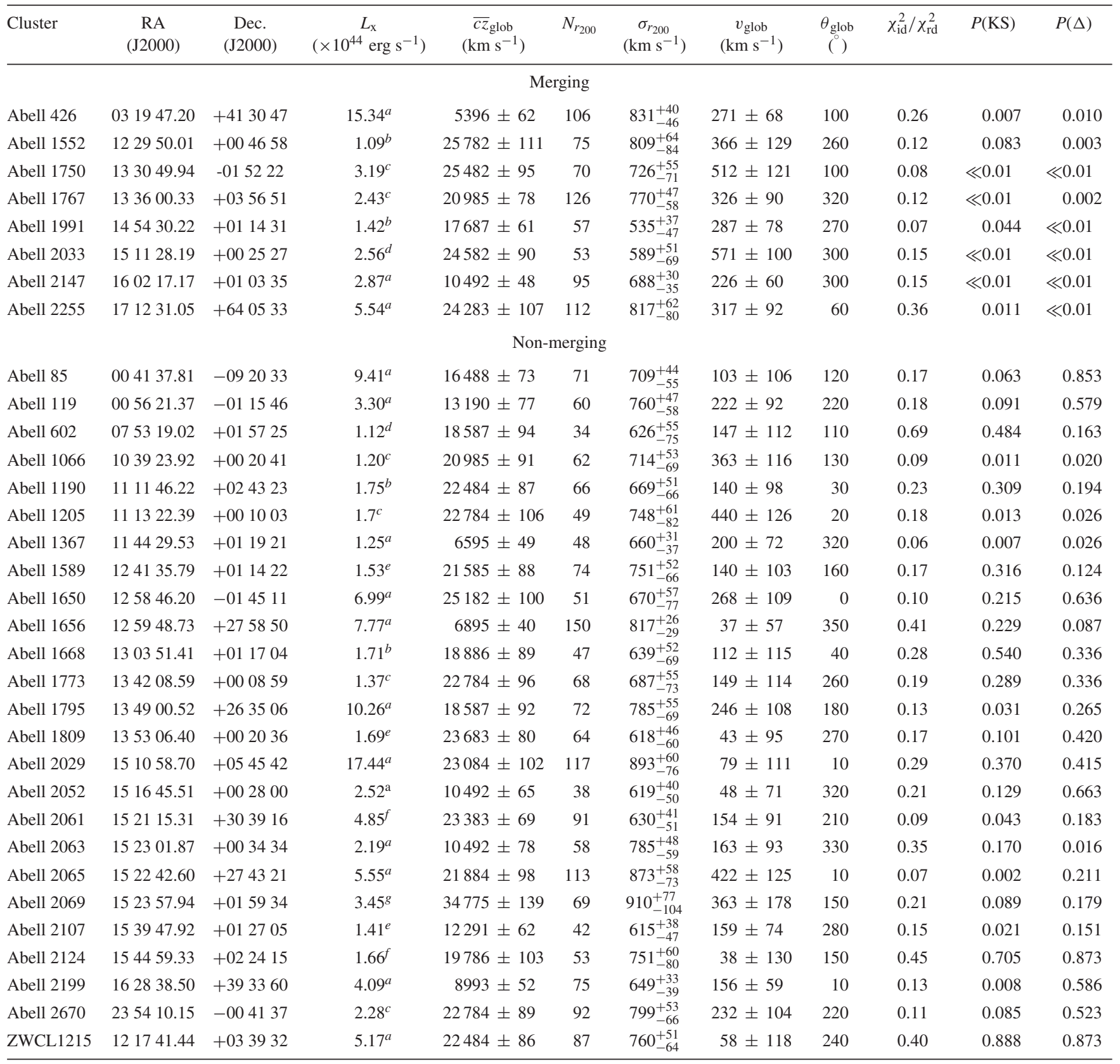

${ }^{a}$ Reiprich \& Böhringer (2002).

${ }^{b}$ Böhringer et al. (2000).

${ }^{c}$ Popesso et al. (2007).

${ }^{d}$ Ebeling et al. (1998).

${ }^{e}$ Jones \& Forman (1999).

${ }^{f}$ Marini et al. (2004).

${ }^{g}$ David, Forman \& Jones (1999).

samples. Following the methodologies of MP17, we determine the ideal $\left(\chi_{\mathrm{id}}^{2}\right)$ and random $\left(\chi_{\mathrm{rd}}^{2}\right) \chi^{2}$ statistic as a by-product of our analysis and can be found within Table 1. Fig. 2 presents an example of the MP17 methodology with our BAX merging and non-merging cluster sub-samples (consistent with the examples in Fig. 1) to determine cluster rotation in the form of the sinusoidal curves produced by artificially rotating the clusters in the plane of the sky. 
Table 2. The volume-limited NED cluster sample as per Pimbblet (2008). The descriptors for the NED cluster kinematic values can be found in Table 1.

\begin{tabular}{|c|c|c|c|c|c|c|c|c|c|c|}
\hline Cluster & $\begin{array}{c}\text { RA } \\
(\mathrm{J} 2000)\end{array}$ & $\begin{array}{l}\text { Dec. } \\
\text { (J2000) }\end{array}$ & $\begin{array}{c}\overline{c z}_{\text {glob }} \\
\left(\mathrm{km} \mathrm{s}^{-1}\right)\end{array}$ & $N_{r_{200}}$ & $\begin{array}{c}\sigma_{r_{200}} \\
\left(\mathrm{~km} \mathrm{~s}^{-1}\right)\end{array}$ & $\begin{array}{c}v_{\text {glob }} \\
\left(\mathrm{km} \mathrm{s}^{-1}\right)\end{array}$ & $\begin{array}{c}\theta_{\text {glob }} \\
\left({ }^{\circ}\right)\end{array}$ & $\chi_{\text {id }}^{2} / \chi_{\text {rd }}^{2}$ & $P(\mathrm{KS})$ & $P(\Delta)$ \\
\hline Abell 533 & 050130.79 & -013027 & $14000 \pm 171$ & 22 & $751_{-153}^{+99}$ & $951 \pm 319$ & 270 & 0.09 & 0.002 & 0.026 \\
\hline Abell 2911 & 012604.60 & -023154 & $24223 \pm 85$ & 31 & $484_{-68}^{+49}$ & $250 \pm 118$ & 50 & 0.19 & 0.058 & 0.129 \\
\hline Abell 3151 & 034027.71 & -015449 & $20265 \pm 115$ & 50 & $753_{-91}^{+67}$ & $278 \pm 168$ & 10 & 0.09 & 0.227 & 0.041 \\
\hline Abell 3266 & 043124.10 & -040547 & $17657 \pm 54$ & 281 & $825_{-38}^{+34}$ & $55 \pm 81$ & 200 & 0.13 & 0.219 & 0.356 \\
\hline Abell 3528 & 125418.20 & -015605 & $15829 \pm 71$ & 103 & $674_{-53}^{+43}$ & $48 \pm 89$ & 140 & 3.61 & 0.663 & 0.834 \\
\hline Abell 3570 & 134652.50 & -023129 & $10972 \pm 50$ & 16 & $233_{-45}^{+30}$ & $110 \pm 81$ & 150 & 1.09 & 0.023 & 0.612 \\
\hline Abell 3535 & 125748.55 & -015357 & $19546 \pm 53$ & 28 & $291_{-43}^{+31}$ & $87 \pm 69$ & 30 & 1.15 & 0.402 & 0.319 \\
\hline Abell 3653 & 195300.90 & -032807 & $32647 \pm 84$ & 43 & $565_{-63}^{+48}$ & $261 \pm 114$ & 170 & 0.10 & 0.094 & 0.115 \\
\hline Abell 3716 & 205116.70 & -033047 & $13850 \pm 76$ & 117 & $767_{-57}^{+47}$ & $200 \pm 110$ & 220 & 0.06 & 0.152 & 0.586 \\
\hline
\end{tabular}
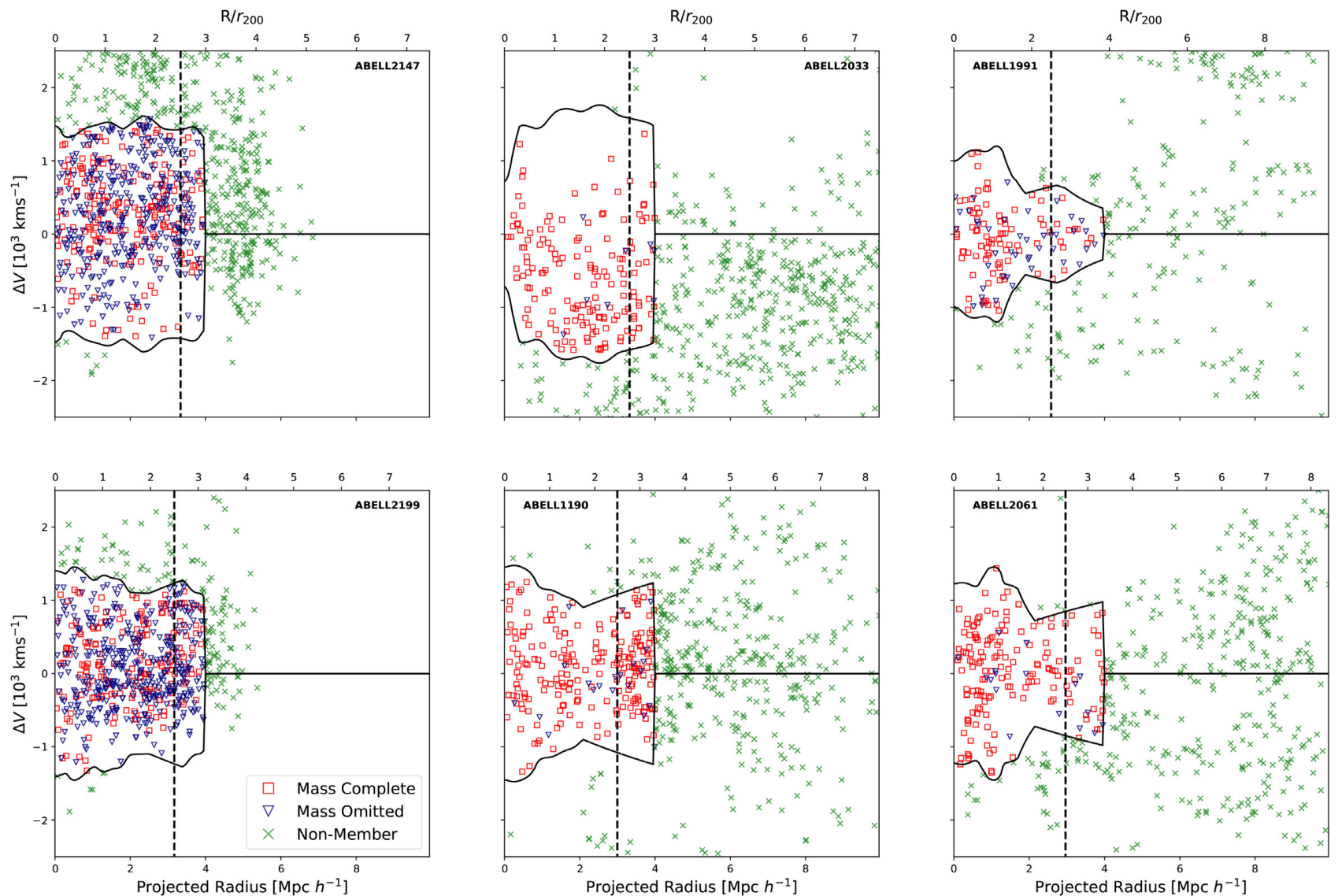

Figure 1. Example surface caustics (the black curves) from the final merging cluster sample (top row) and non-merging cluster sample (bottom row). The red squares represent the galaxies that make a complete sample at $\log _{10}\left(\mathrm{M}_{*}\right) \geq 10.2$; the blue triangles represent omitted galaxies that are at $\log _{10}\left(\mathrm{M}_{*}\right)<10.2$. Galaxies that lie within the surface caustics are considered to be cluster members. Here the radial velocity $(\Delta V)$ with respect to the cluster's mean recession velocity is plotted against the projected radius in units of $\mathrm{Mpc} h^{-1}$ and $R / r_{200}$. The black dashed vertical lines represent the $2.5 R / r_{200}$ radial cut of each cluster; galaxies $\leq 2.5 R / r_{200}$ within the caustics produce the rotational profile stacks.

The thesis presented here is focused on how $v_{\text {diff }}$ is dependent on the cluster galaxy sub-populations as a function of cluster radius at different epochs; core-merging events between two originally independent clusters; substructuring of galaxies relaxing from a core-merger event; older and relaxed clusters that are homogeneous to our tests of substructure. We therefore, using the calculated global cluster defined values, determine how $v_{\text {glob }}$ varies as a function of radius from the cluster centre in incremental units of $0.1 r_{200}$ with a coverage of $0<R \leq 2.5 r_{200}$ by fixing our theta to the rotational axis $\theta_{\text {glob }}$. An example of the application of this methodology to the individual BAX-defined clusters between merging and non-merging environments, as defined previously by the $\Delta$-test, is depicted in Fig. 3. 

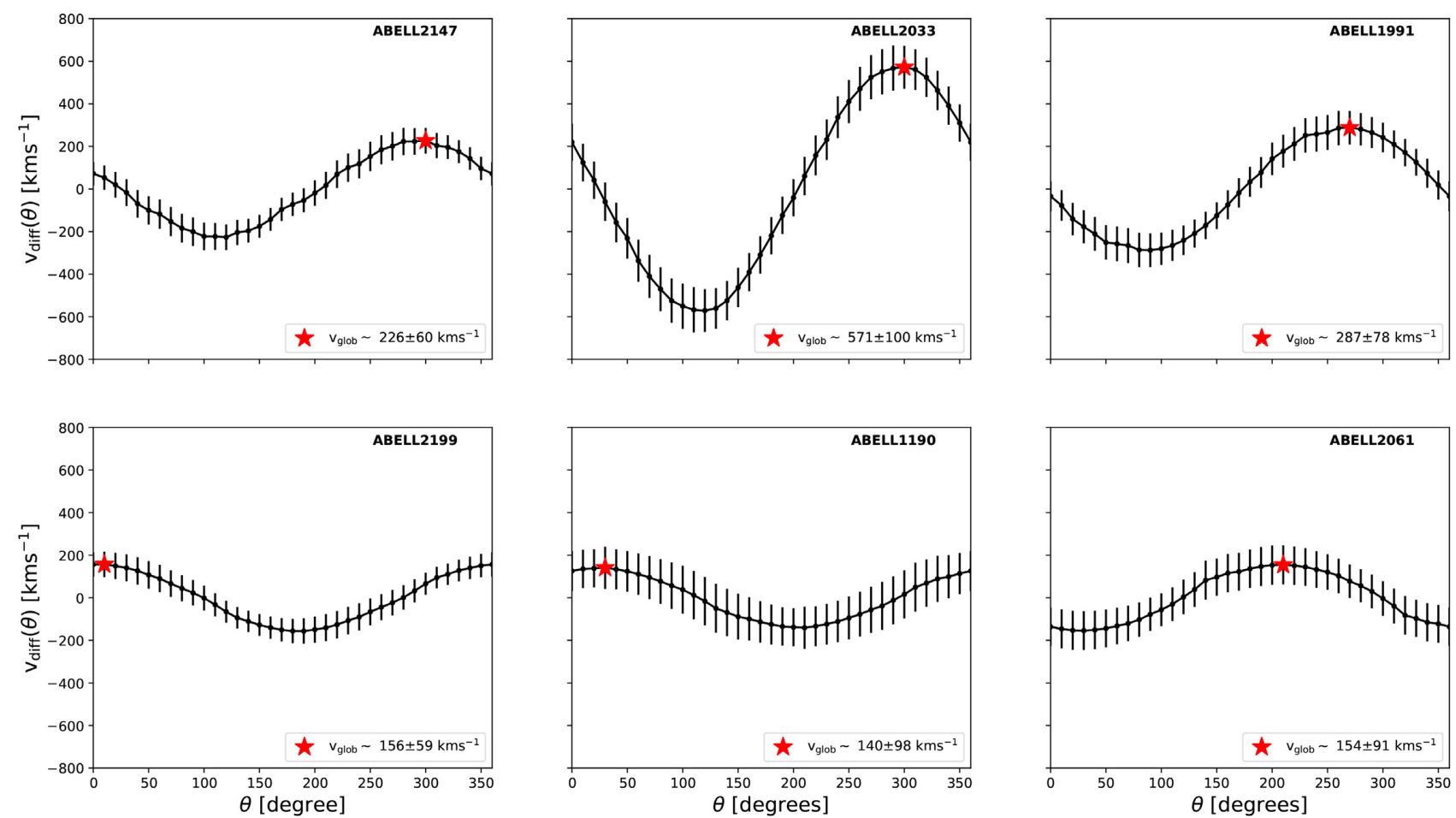

Figure 2. A selection of example BAX cluster sinusoidal rotational curves of merging (top row) and non-merging (bottom row) clusters, as determined by the $\Delta$-test for substructure with galaxies that lie $\leq 1.5 \mathrm{Mpc} h^{-1}$ from the cluster centre, with $v_{\text {diff }}$ as a function of $\theta$ as per the MP17 methodology outlined in Section 3.2. The red star marks the point at which $v_{\text {glob }}=\operatorname{MAX}\left[v_{\text {diff }}(\theta)\right]$. The uncertainties on the real data curve are derived by the propagation of the standard error as denoted in equation (4).

Here we find the merging clusters demonstrate rising profiles from the cluster centre that lead to consistently high $v_{\text {diff }}\left(\theta_{\text {glob }}\right)$ values throughout to $2.5 r_{200}$. In contrast, non-merging clusters possess dampened core-rotational velocities, with Abell 2199 showing a consistent profile out towards $2.5 r_{200}$, most likely as a result of the outer galaxy members homogenizing with the cluster's angular momentum. The behaviour observed here between the two subsamples runs parallel to the $\Delta$-test for substructure; increasing corerotational velocities show correlation with merging environments. This response of environment to the rotational velocities is not completely surprising considering both methodologies are constrained to the same projected radii and radial velocity measurements. It should be noted that this effect is not completely consistent to every $\Delta$-test-defined merging cluster, which highlights the limitations of analysing 3D motions through a projected 2D-plane of sky.

\subsection{Dumbbell BCG clusters}

If we assume that the evolution of angular momentum within clusters originates from the off-axial interaction between two smaller clusters, then this could potentially be detected through lineof-sight measurements that are sensitive to determining rotation. Hence, we consider clusters that host multiple BCG components with significant velocity offsets which could be the result of recently merged sub-clusters that are relaxing on to a common potential. Therefore, we elected to study a sample of dumbbell BCG clusters for their global rotational profiles with the aim to test if their offset peculiar velocity BCG cores are an indicator of higher levels of merger activity, resembling earlier epochs of post-merger relaxation. Using the volume-limited sample as outlined in Section 2 with the NED galaxies we perform the $\Delta$-test for substructure from the NED centres to $\leq 1.5 \mathrm{Mpc} h^{-1}$ (see 3.1). A comparison between the statistical results, alongside the bubble plots, and the global rotational profiles, as determined in Section 3.2, of each dumbbell BCG cluster is made. An example of these results for our dumbbell BCG hosting clusters can be found in Figs 4, 5, and 6.

We, unsurprisingly, do not find a significant correlation between substructuring and the presence of multiple offset velocity BCGs, consistent with the findings of Pimbblet (2008). This is despite the use of a standard, more loose, criteria where in this work substructure is deemed significant at $P(\Delta) \leq 0.01$. It is more likely that substructure would not play a key role in the instance of dumbbell cluster BCG cores due to the collisionless nature of galaxies on the time-scales presented, especially if the dumbbell cores are in the early stages of a merger between two initially independent potential wells. Abell 3391 is the only cluster in the dumbbell sample found to possess substructure; the bubble plot is presented, along with the rotational profile, in Fig. 4. It is interesting to note how the rotational profile of Abell 3391 decreases to a minimum at $\sim 1.0 \mathrm{Mpc} h^{-1}$, before rising back to previous $v_{\text {glob }}$ values within the same projected radial separation. This, compared with the substantial substructuring observed with the bubble plot, illustrates a strong double-component system of rotating galaxies; a fast-rotating core and a fast-rotating outer region as a result of an ongoing active merging event between two originally independent BCGs and their host galaxy clusters. This inference is exacerbated by comparing the same analysis the dumbbell hosting clusters of Abell 3716 and Abell 3653, which can be seen in Figs 5 and 6, respectively. The $v_{\text {glob }}$ profile of Abell 3716 in particular marries closely to that of Abell 3391, decreasing to a minimum $\lesssim 1.0 \mathrm{Mpc}$ $h^{-1}$, before only subtly rising back to dampened levels of rotation where $v_{\text {glob }} \sim 200 \mathrm{~km} \mathrm{~s}^{-1}$. From the bubble plot of Abell 3716 

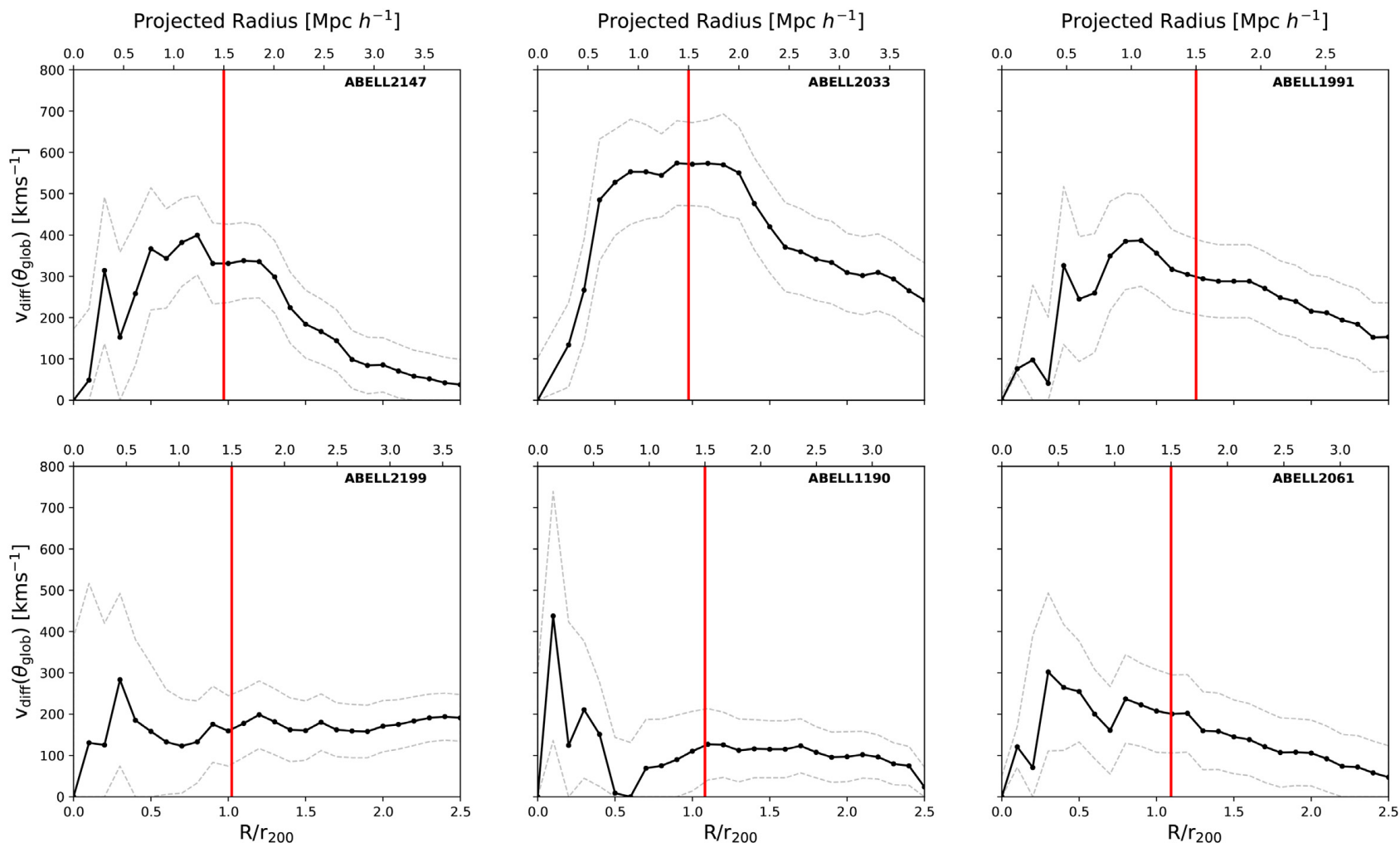

Figure 3. A selection of example $v_{\text {diff }}\left(\theta_{\text {glob }}\right)$ rotational profiles consistent with Fig. 2, as a function of the projected virial radius $R / r_{200}$ in increments of 0.1 , of merging (top row) and non-merging (bottom row) as determined by the $\Delta$-test for substructure. The red vertical lines represent the point at which the global cluster values are determined and the statistical tests for both substructure and rotation are calculated with galaxies that $\leq 1.5 \mathrm{Mpc} h^{-1}$ from the cluster centre. Note the consistency of higher rotational velocity throughout the merging clusters in comparison to the dampened profiles for the non-merging clusters. The dashed lines represent the uncertainties derived from the propagated standard error as denoted in equation (4).

we can see there are some small pockets of deviation from the global values, although not to the levels found in Abell 3391. We can therefore surmise that the Abell 3391 and 3716 are dumbbell hosting clusters in different stages of merging, where the Abell 3391 is in an active phase of merging with intense galaxy-galaxy interactions providing off-axial angular momentum donation, and Abell 3716 in an earlier, less-active phase of merging, where the galaxies are still yet to interact due to their collisionless nature. Abell 3653 presents a $v_{\text {glob }}$ profile with a remarkably consistent zero gradient with exception of the bulk increase in rotation at 0.5 Mpc $h^{-1} \lesssim R \lesssim 1.0 \mathrm{Mpc} h^{-1}$. It is also notable how the bubble plot of Abell 3653 has overt displays of substructure towards the west of the sky, where aside from the sizeable peculiar velocity of the BCG addressed in the study of Pimbblet et al. (2006), X-ray analysis conducted by Caglar \& Hudaverdi (2017) has shown the location of this substructure to be coincident with another BCG hosting sub-cluster. As further stated within the work of Caglar \& Hudaverdi (2017), the presence of harder X-ray emission in the space in between these two independent BCGs, along with their $\sim 35 \mathrm{kpc}$ offset from their respective $\mathrm{X}$-ray peaks, is a shock region between their ICM environments indicative of an ongoing initial merger phase. Taking into account the projected radial separation between the two BCGs of these sub-clusters $(\sim 500 \mathrm{kpc})$, we can see that the bulk rotation observed $0.5 \mathrm{Mpc} h^{-1} \lesssim R \lesssim 0.8 \mathrm{Mpc}$ $h^{-1}$ is primarily the result of the foreground BCG towards the west of the sky combined with host sub-cluster's members, as can be seen by the apparent dichotomy in the radial velocities. The clear background and foreground structures, along with the even delineation between them, throughout the projected radius studied creates the impression of a consistent $v_{\text {glob }}$ profile, aside from the boost provided by substructured west BCG $\sim 0.5 \mathrm{Mpc} h^{-1}$, which introduces the possibility that the global rotation via our method is merely the result of the z-space difference between the two sub-clusters. With this in mind, however, Caglar \& Hudaverdi (2017) have concluded the sub-clusters are gravitationally bound and are in infall at $2400 \mathrm{~km} \mathrm{~s}^{-1}$ with core passage expected in 380 Myr. The peculiar velocities of the dumbbell components of each cluster shown here, along with the two independent Abell 3653 BCG components, are detailed in Table 3 for easy comparison. Considering the results from the examples shown here the varying rotation profile, close angular separation between the cores and levels of substructuring present in Abell 3391, the cluster must be in a 'post-initial merger' phase; the two cores are relaxing on to a common potential with the surrounding population of galaxies aggressively interacting with one another as a result of their latent friction and global rotation donated from the initial merging phase.

\subsection{BAX cluster stacks}

In order to test the dynamical evolution of clusters more generally, we make attempts to observe any contrast in the global rotation profiles across differing cluster environments that could represent different epochs of cluster-cluster merging. Therefore, to build this general picture, we build composite clusters from the BAX sample between those defined as either merging or non-merging, which has the primary benefit of boosting the signal to noise for the rotational profiles. For the purposes of calculating $v_{\text {rot }}$ using MP17, following 

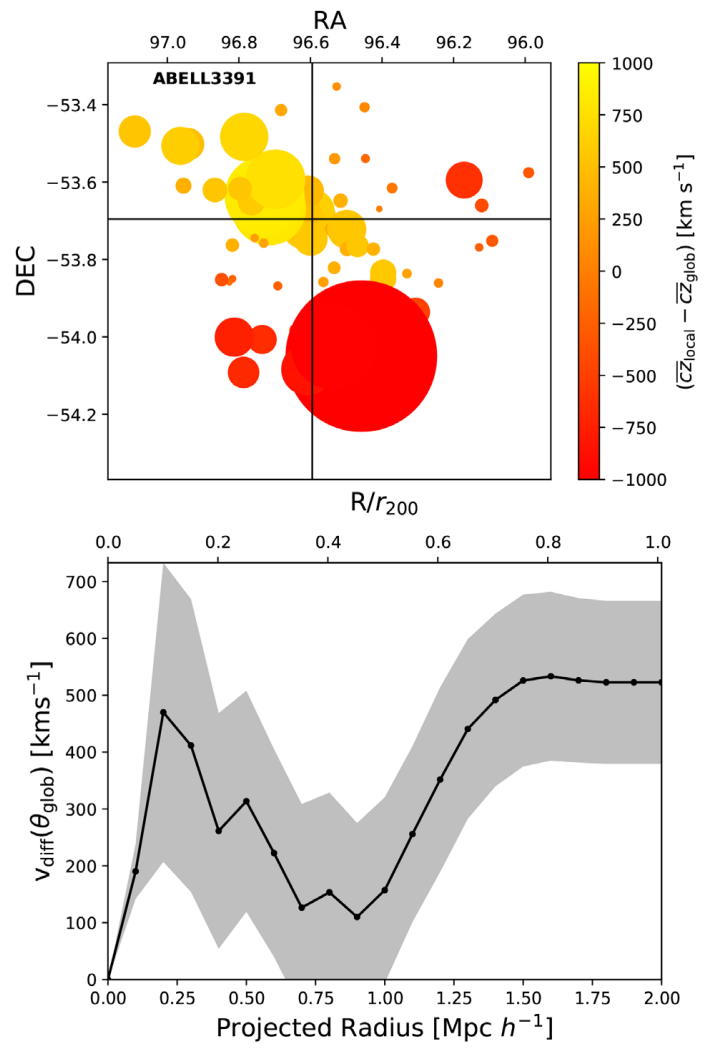

Figure 4. Top: bubble plot of Abell 3391 from the $\Delta$-test, where the size of each circle is proportional to $\pi\left(e^{\delta_{i}}\right)^{2}$; the black cross represents the NEDdefined centre and the colours represent varying radial velocity differences $\left[\overline{c z}_{\text {local }}-\overline{c z}_{\text {glob }}\right]$. Bottom: the rotational profile of Abell 3391.

the outlined procedure in Section 3.2, we initiate the following stacking procedure: each cluster is rotated by their respective $\theta_{\text {glob }}$ so the rotation axis of each cluster overlaps, and then we stack our clusters on to a common RA-Dec. grid normalized to each cluster's respective BAX centres along with their normalized $\Delta \mathrm{V}$ values as per equation (3). This will lead to the rotational axis of each composite stack becoming $\sim 0^{\circ}$, which provides, $v_{\text {rot }}=v_{\text {diff }}(\theta=$ 0 ). The final galaxy contributions to the merging and non-merging stacks are 1286 and 3349 galaxies, respectively.

The first result of the complete merging and non-merging composites are highlighted for comparison in Fig. 7. We can immediately see that the rotational profile of the merging composite in Fig. 7 possesses very high core rotation peaking up to $\sim 400 \mathrm{~km} \mathrm{~s}^{-1}$ within $\lesssim 0.5 R / r 200$. This result is indicative of the merging sub-sample primarily consisting of relaxing galaxy cluster cores that have undergone recent core-merging processes. The continued high $v_{\text {rot }}$ retained throughout to $\lesssim R / r_{200}$ implies this angular momentum donation mechanism is dominant. The gradual decline in $v_{\text {rot }}$ at $\gtrsim R / r_{200}$ is the result of a decrease in cluster galaxy density, and therefore, interaction probability between them. Contrarily the rotational profile of the non-merging composite presents a dampened core rotation. The immediate inference of this dampening effect presents the non-merging composite to mainly consist of older, more evolved, clusters in more advanced stages of relaxation processes. However, there is a need to consider that the differences in our observations of $v_{\text {rot }}$ between the merging and non-merging galaxies are down to their potential difference in mass distributions.
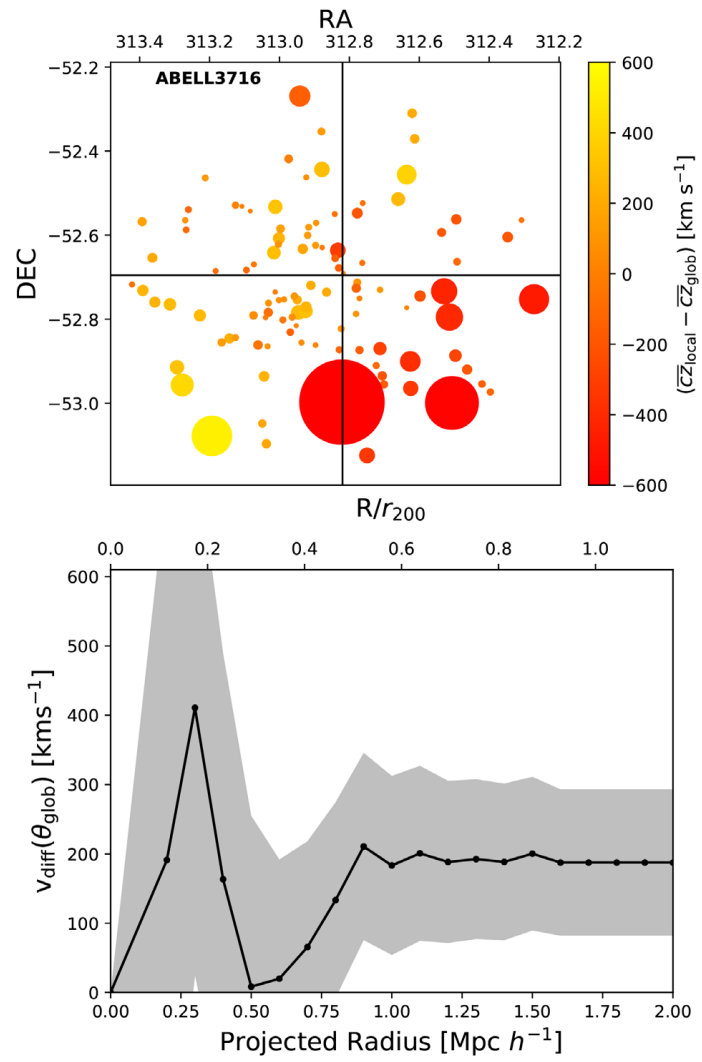

Figure 5. Top: bubble plot of Abell 3716 from the $\Delta$-test, where the size of each circle is proportional to $\pi\left(e^{\delta_{i}}\right)^{2}$; the black cross represents the NEDdefined centre and the colours represent varying radial velocity differences $\left[\overline{c z}_{\text {local }}-\overline{c z}_{\text {glob }}\right]$. Bottom: the rotational profile of Abell 3716.

To see how cluster galaxy colour sub-populations respond to the dynamics, activity, and environment, the galaxies for the merging and non-merging composites are split into two sub-populations of colour: blue galaxies and red galaxies. In order to account for the biasing of colour distributions with increasing galaxy log stellar mass we find a line of delineation that determines a galaxy's colour, which is computed with a $(u-r)$ colour gradient as a function of the log stellar mass. Following the methodology of Jin et al. (2014), the residual galaxies from the bimodal $(u-r)$ distribution in bins of increasing stellar mass are used to output the $k$-corrected linear relation $(u-r)_{z=0}=0.40\left[\log _{10}\left(M_{*}\right)\right]-1.74$, this is further detailed in equation (4) of Bilton \& Pimbblet (2018).

All BAX clusters with their member galaxies holding DR8 photometry are $k$-corrected to the local rest frame $(z=0)$ before being parsed through the linear relation denoted above. Galaxies that lie at greater values from the linear gradient are all classified as red-sequence galaxies, with galaxies below classified as blue cloud galaxies. An example of the colour distributions of the galaxies for each stack can be found in Fig. 8, providing the merging sample with 402 blue galaxies and 862 red galaxies alongside the non-merging sample with 1153 blue galaxies and 2184 red galaxies for each stack. The $v_{\text {rot }}$ profiles are then calculated for each sub-population, and environment, utilizing the same stacking and outputting sequence highlighted previously in the production of Fig. 7. Fig. 9 presents the resultant $v_{\text {rot }}$ profile with the above implemented methodology. The merging rotational profile in Fig. 9 depicts the blue sub-population of galaxies with very high segregation of $v_{\text {rot }}$ values at $\lesssim R / r 200$. However, due to a depletion 

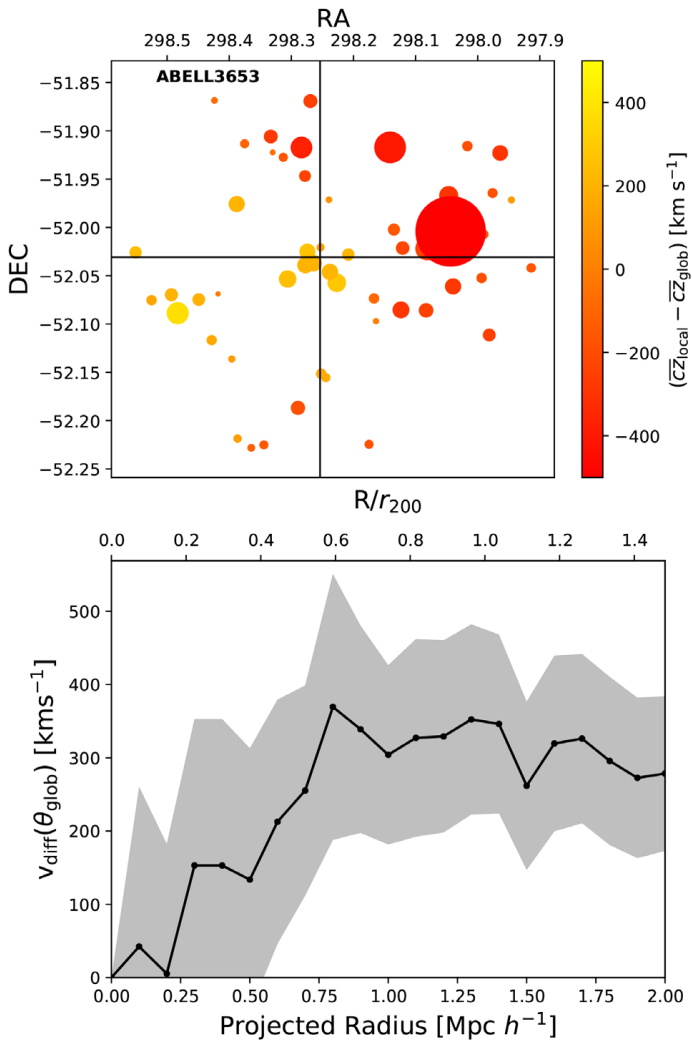

Figure 6. Top: bubble plot of Abell 3653 from the $\Delta$-test, where the size of each circle is proportional to $\pi\left(e^{\delta_{i}}\right)^{2}$; the black cross represents the NEDdefined centre and the colours represent varying radial velocity differences $\left[\overline{c z}_{\text {local }}-\overline{c z}_{\text {glob }}\right]$. Bottom: the rotational profile of Abell 3653.

Table 3. The peculiar velocities of the example dumbbell hosting clusters presented in Figs 4, 5, and 6 within the reference frames of their respective $\overline{c z}$ values, referenced from Pimbblet (2008). The literary values for the BCGs of Abell 3653 are utilized from Caglar \& Hudaverdi (2017).

\begin{tabular}{rccc}
\hline $\begin{array}{l}\text { Cluster } \\
\text { components }\end{array}$ & $\begin{array}{c}\text { RA } \\
(\mathrm{J} 2000)\end{array}$ & $\begin{array}{c}\text { Dec. } \\
(\mathrm{J} 2000)\end{array}$ & $\begin{array}{c}|\Delta c z| \\
\left(\mathrm{km} \mathrm{s}^{-1}\right)\end{array}$ \\
\hline $\begin{array}{c}\text { Abell 3391 DBL1 } \\
\text { DBL2 }\end{array}$ & 062620.22 & -531457.84 & $489 \pm 133$ \\
Abell 3653 DBL1 & 195303.48 & -531456.04 & $68 \pm 142$ \\
DBL2 & 195302.76 & -520806.00 & $495 \pm 126$ \\
BCG1 & 195301.90 & -525913.00 & $683 \pm 96$ \\
BCG2 & 195217.30 & -515950.00 & $43 \pm 124^{a}$ \\
Abell 3716 DBL1 & 205200.48 & -521618.48 & $559 \pm 92$ \\
DBL2 & 205166.88 & -521615.60 & $255 \pm 88$ \\
\hline
\end{tabular}

${ }^{a}$ The uncertainty is propagated through from the literary values.

of blue galaxies towards the core there are no $v_{\text {rot }}$ values for the blue sub-population $\lesssim 0.3 R / r_{200}$. Consequentially, this implies the observed core rotation from the merging composite in Fig. 7 is dominated by red-sequence galaxies. The immediate conclusion as a result implies that cores of merging clusters consist of evolved red galaxies in the process of relaxing on to a new common cluster potential via 'back and forth' sloshing motions. The non-merging profile in Fig. 9 demonstrates a tighter velocity separation between the blue and red galaxy sub-populations, however, there is still a clear segregation in rotational velocity that leads to the connotations of infalling blue galaxies. There is the significant 'bump' in the blue galaxy sub-population at $\sim R / r_{200}$, which could inconclusively be the result of a mixture of infaller and so-called 'backsplash' galaxies within the stack (see Pimbblet 2011). The key result from the $v_{\text {rot }}$ composite profiles of the colour sub-populations is that the two environments are indicative of differing epochs of cluster merging; relaxed galaxies with a population of infalling, or potentially backsplash, blue-galaxies within the non-merging composite and an actively relaxing galaxies on to a common potential from successive merging processes depicting the merging composite.

We show the $L_{X}-z$ distribution for each BAX sub-sample with comparison to the downloaded BAX catalogue in Fig. 10 as a proxy for mass distributions present within our BAX sample. Despite some outliers from the non-merging sample, we can see that both the merging and non-merging samples inhabit comparable mass distributions within similar redshifts. However, we briefly test how sensitive the rotational profile composites are to the evolutionary epochs and masses by constraining our BAX samples to those that fall within the redshift range of $0.03 \leq z<0.09$ and X-ray luminosity values of $<6 \times 10^{44} \mathrm{erg} \mathrm{s}^{-1}$, this implementation results in a tighter parity between the two sub-samples. Figs 11 and 12 illustrate the constrained sample $v_{\text {rot }}$ composites.

Despite the tighter constraint, the full composites presented in Fig. 11 are similar to the unconstrained composite in Fig. 7, with exception of a dampened core in the merging profile and a general shift in the magnitude of $v_{\text {rot }}$ in both merging and nonmerging composites. The most notable difference is found with the constrained colour composite in Fig. 12 where the blue subpopulation of the non-merging stack is subdued with $v_{\text {rot }}$ values falling below the red sub-population within the core regions at $\lesssim r_{200}$. The constrained non-merging composite suffers large drops in galaxy numbers contributing to the analysis that leaves 721 blue galaxies and 11365 red galaxies. The general shape of the profile itself is retained with dampened $v_{\text {rot }}$ values as has been consistently shown, with the only notable significant loss found at $\sim r_{200}$ with the peak of the retained 'bump' shape of the blue sub-population homogenizing with the red sub-population. This knowledge, alongside the high uncertainties of the non-merging blue sub-population overlapping with the relatively unaffected red sub-population, indicates that the drop in the rotational velocities within the core regions is not significant in displaying a different picture of non-merging systems as shown in Fig. 9. This is aided by considering the large omission of non-merging clusters for the constrained composites; it is therefore no surprise that the nonmerging blue sub-population is more sensitive to the constraints, which is especially the case within the core regions where the number of blue galaxies are fewer. Furthermore, it has already been noted how the shape of the blue sub-population in the non-merging sample is consistent, in addition to the overlapping uncertainties, which implies that this is predominantly a signal-noise problem.

\section{SIMULATING THE TRANSVERSE MOTIONS}

In this section, we describe a 3D simulation of an idealized binary major cluster merger and evaluate how the merger process affects the rotation of the resultant system. We look in particular at how merger phase and viewing angle changes the rotation rate when viewed in an observer-like 2D projection and attempt to draw parallels to the observations.

The simulation is built upon the FLASH code, a publicly available high-performance modular code (Fryxell et al. 2000), utilizing the 3D hydrodynamic $+N$-body capabilities to simulate the gaseous ICM and collisionless dark matter (DM), respectively. Both components being self-gravitating allows the effects of dynamical friction 

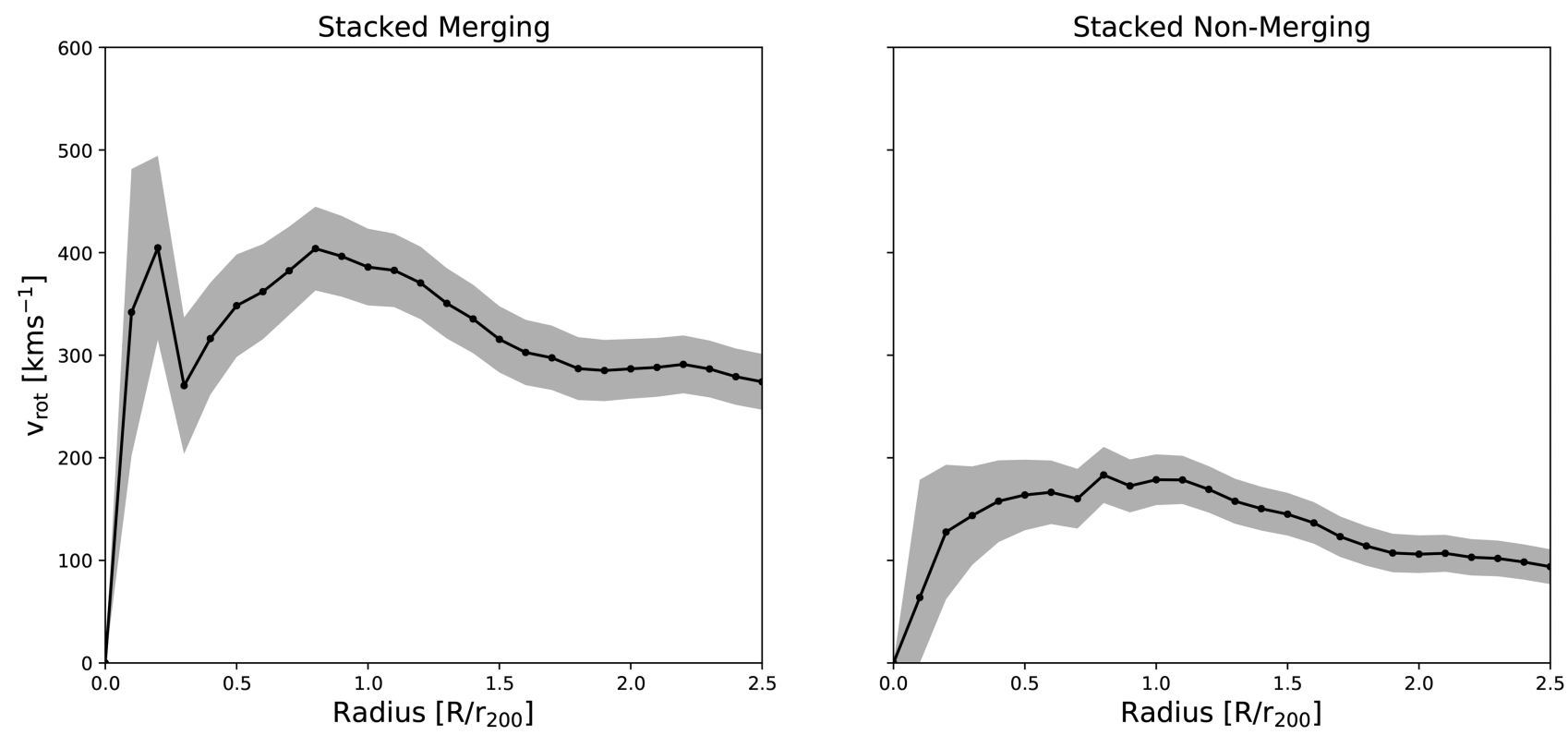

Figure 7. Composite rotational velocity profiles of all galaxies as a function of radius $\left(R / r_{200}\right)$. The merging rotational profile (left) displays a high rotational velocity at the core $\leq 0.5 R / r_{200}$. In contrast the non-merging rotational profile (right) shows dampened core rotation, which collapses close to zero at radii $\geq 0.5 R / r_{200}$. The shaded regions represent the uncertainties derived from the propagated standard error as denoted in equation (4).
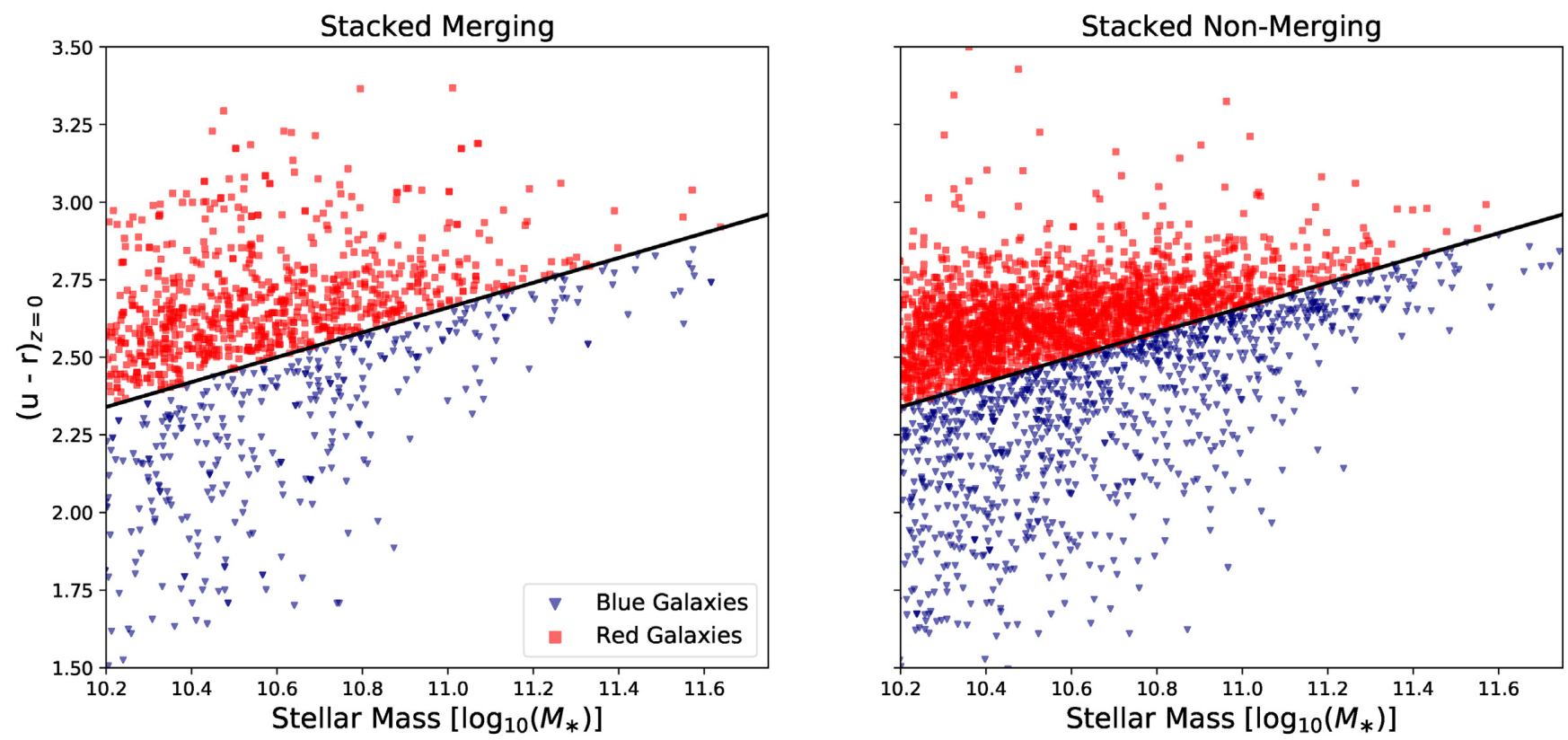

Figure 8. The colour distributions of the mass-complete DR8 galaxies between the merging and non-merging samples: $(u-r)_{z}=0$ plotted as a function of $\log _{10}\left(M_{*}\right)$. The black line resembles the linear fit of the centre of the bimodal distribution at quartile increments of $\log _{10}\left(M_{*}\right)$; red galaxies are above the fitted line denoted as red squares; blue galaxies are below the fitted line denoted as blue triangles.

and tidal forces to be captured in the simulation. Taking advantage of the adaptive mesh capabilities of FLASH and refining on particle density results in a maximum resolution in the cluster cores of $19.6 \mathrm{kpc}$.

The simulation consists of a 1:2 cluster merger with masses of $5 \times 10^{14}$ and $1 \times 10^{15} \mathrm{M}_{\odot}$ and $r_{200}$ values of 1672 and $2107 \mathrm{kpc}$, respectively. Following the set-up procedure described in ZuHone (2011), both clusters are non-rotating cool-core clusters possessing spherically symmetric single Hernquist mass profiles (Hernquist 1990 ) with a $\beta$-profile for the ICM density. The bulk of the mass is provided by 3 million and 6 million DM particles in the smaller and larger cluster, respectively. The initial conditions are set such that at the point the two $r_{200}$ cross one another the relative cluster velocity is $1.1 V_{\mathrm{c}}$ (where $V_{\mathrm{c}}=\sqrt{G M_{\mathrm{vir}} / r_{\mathrm{vir}}}$ ), in accordance with the average infall velocity on to a cluster found from cosmological simulations by Tormen (1997) and Vitvitska et al. (2002). Following Poole et al. (2006), we use a tangential velocity component equal to $0.25 \mathrm{~V}_{\mathrm{c}}$.

To achieve the aims of this section we make the assumption that the simulation's DM particles possess similar motions to that of the galaxies with in the cluster, given that motions of both are collision- 

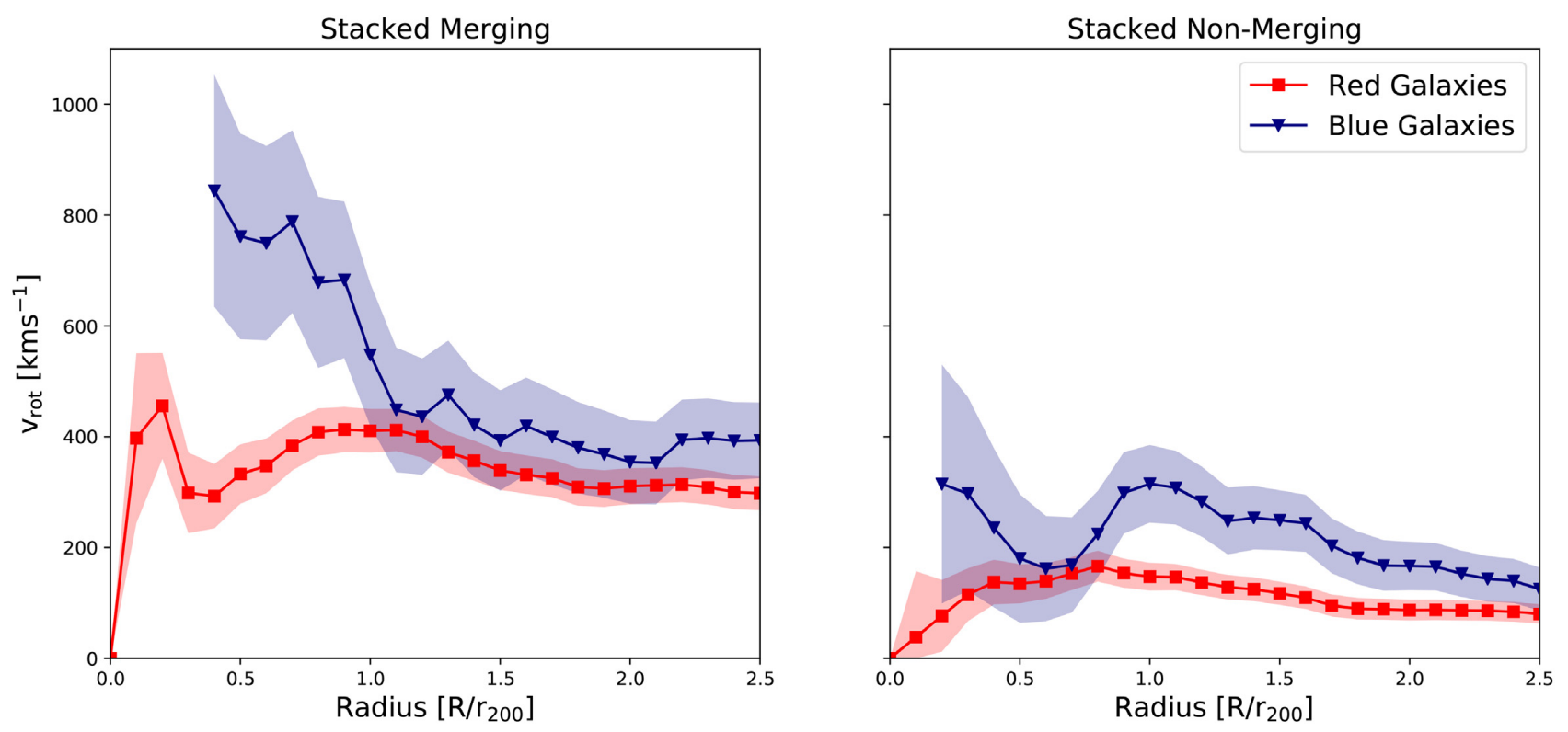

Figure 9. Composite rotational velocity profiles split by their colour with the same axes as Fig. 7. The blue triangle and the red square markers of each stack represent the blue and the red galaxies, respectively. The blue galaxies in the merging cluster stack (left) have a high $v_{\text {glob }}$ segregation from the red galaxies at $0.4 \lesssim R / r_{200} \lesssim 1.0 r_{200}$ before homogenizing $r_{200}$. The non-merging cluster stack (right) observes dampened behaviours with 'bumps' $\gtrsim r_{200}$. The shaded regions represent the uncertainties derived from the propagated standard error as denoted in equation (4).

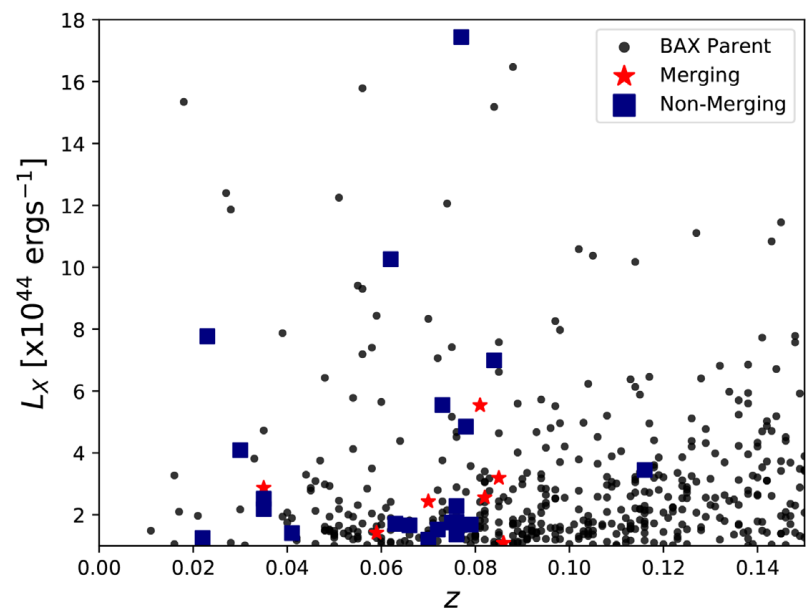

Figure 10. The distribution of BAX cluster $X$-ray luminosities $\left(L_{X}\right)$ against redshift $(z)$. The red stars resemble the merging sample and the blue squares depict the non-merging sample.

less and only feel the effect of dynamical friction. Galaxies would experience higher dynamical friction than a single DM particle given the particles lower mass, however we find that the difference is negligible in this context. Making this assumption allows us to treat the DM particles as galaxies and use their line-of-sight velocities to calculate the radial rotation rate of the cluster using the same method described previously for the observational data.

Fig. 13 shows the evolution of projected DM in the simulation. From this we can see that after first core passage each cluster core looses all of its tangential velocity relative to the second cluster. This is as a result of the significant dynamical friction the two cores experience traversing one another. Consequently, all future infalls of the two cores proceed along a straight path that links the two first apocentres. This linear motion has the property of always being perpendicular to the axis of rotation of the merger. The cluster cores oscillate for roughly $2.5 \mathrm{Gyr}$ after second core passage in which time they pass through one another six times, after which they become indistinguishable from one another and hence have merged. If we make the assumption that the BCGs of each cluster remain at the bottom of their respective potential wells throughout the merger then the previous statements regarding the motions of cluster cores can also be considered true for the dumbbell BCGs.

Fig. 14 shows the radial rotation for the cluster throughout, second passage, from different viewing angles. From this we see that changing the line of sight significantly alters the effect that second infall has on the measured radial rotation rate of the system. Those viewing angles offset from the linear motion of the cluster cores/dumbbell BCGs (A and C in Fig. 14) display dramatic changes in the rotation, dropping from maximum to minimum values within one $r_{200}$ and then to increase again at larger radii. Conversely those parallel or perpendicular (B or D in Fig. 14, respectively) posses far more consistent profiles. The reason for these differences is how the linear motion of the two cores is interpreted via our method of measuring rotation through line-of-sight motion.

If the merger is viewed such that the line of sight is parallel to the linear motion of the dumbbell BCGs (as with row B) then in the bulk linear motion will average to zero due to the symmetry of the overlapping cores, resulting in it not contributing to the rotation profile. Similarly, a line of sight perpendicular to the linear dumbbell motion would be unable to detect the bulk velocities of the cores due to no fraction of their motion being down the line of sight, this again results in no 'peculiar' increase in rotation while still observing the cluster rotation (provided the line of sight was not parallel to the rotation axis where the rotation would not be observable) as can be seen in row D in Fig. 14.

For mergers viewed with an offset from that of the linear motion of the cluster cores/dumbbell BCGs, such as with rows A and C in Fig. 14, the linear motion of the cores is incorporated into the radial rotation. This is due to a substantial component of the velocity of the linear motion being along the line of sight, along with the lack of symmetry the projected system possesses, thus resulting in 

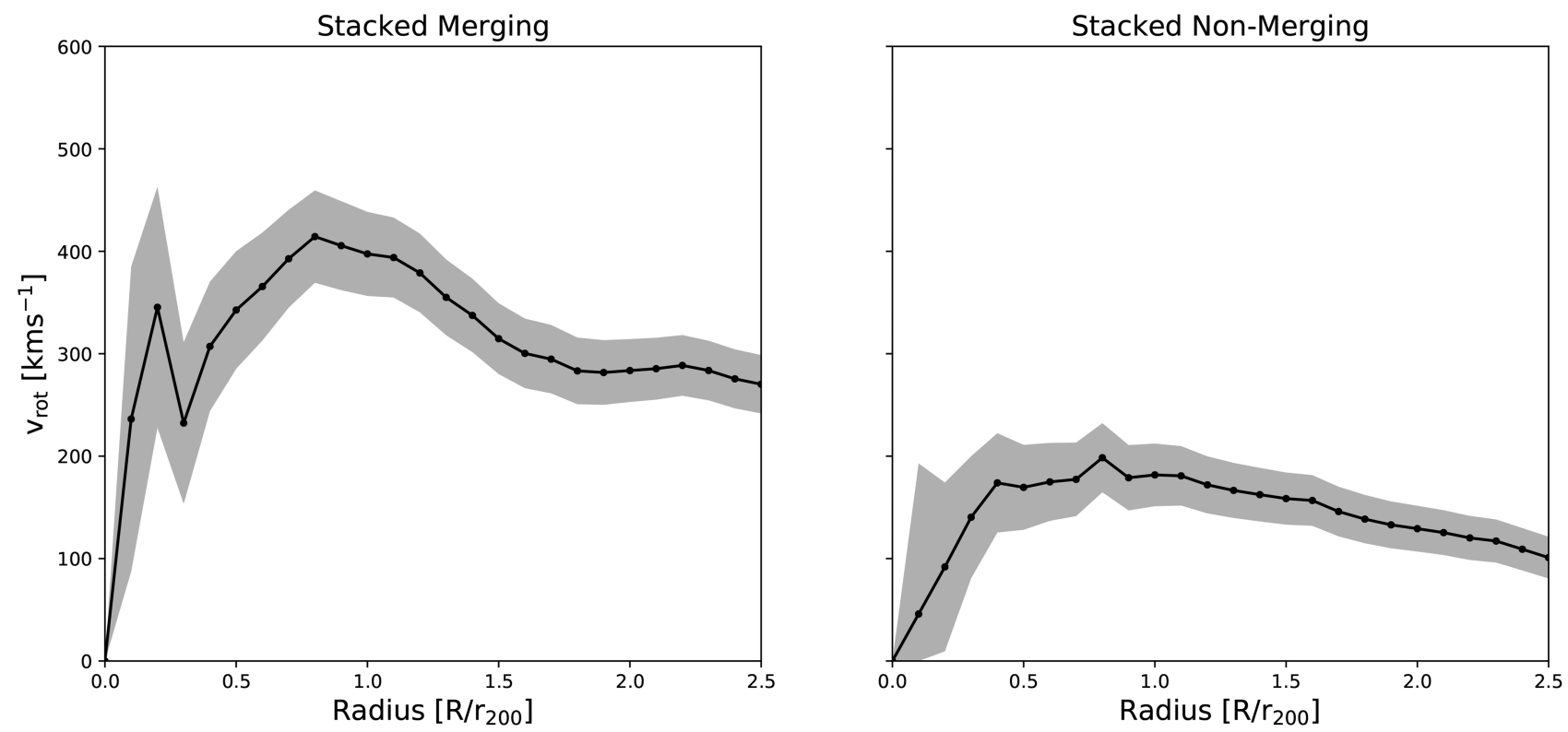

Figure 11. Constrained composite rotational profiles, similar to Fig. 7, with only BAX clusters lying within redshifts of $0.03 \leq z<0.09$ and possessing X-ray luminosities in the range $<6 \times 10^{44} \mathrm{erg} \mathrm{s}^{-1}$. The overall shape of each of the profiles is retained with some shifts in the magnitude of $v_{\text {rot }}$ across both stacks. The shaded regions represent the uncertainties derived from the propagated standard error as denoted in equation (4).
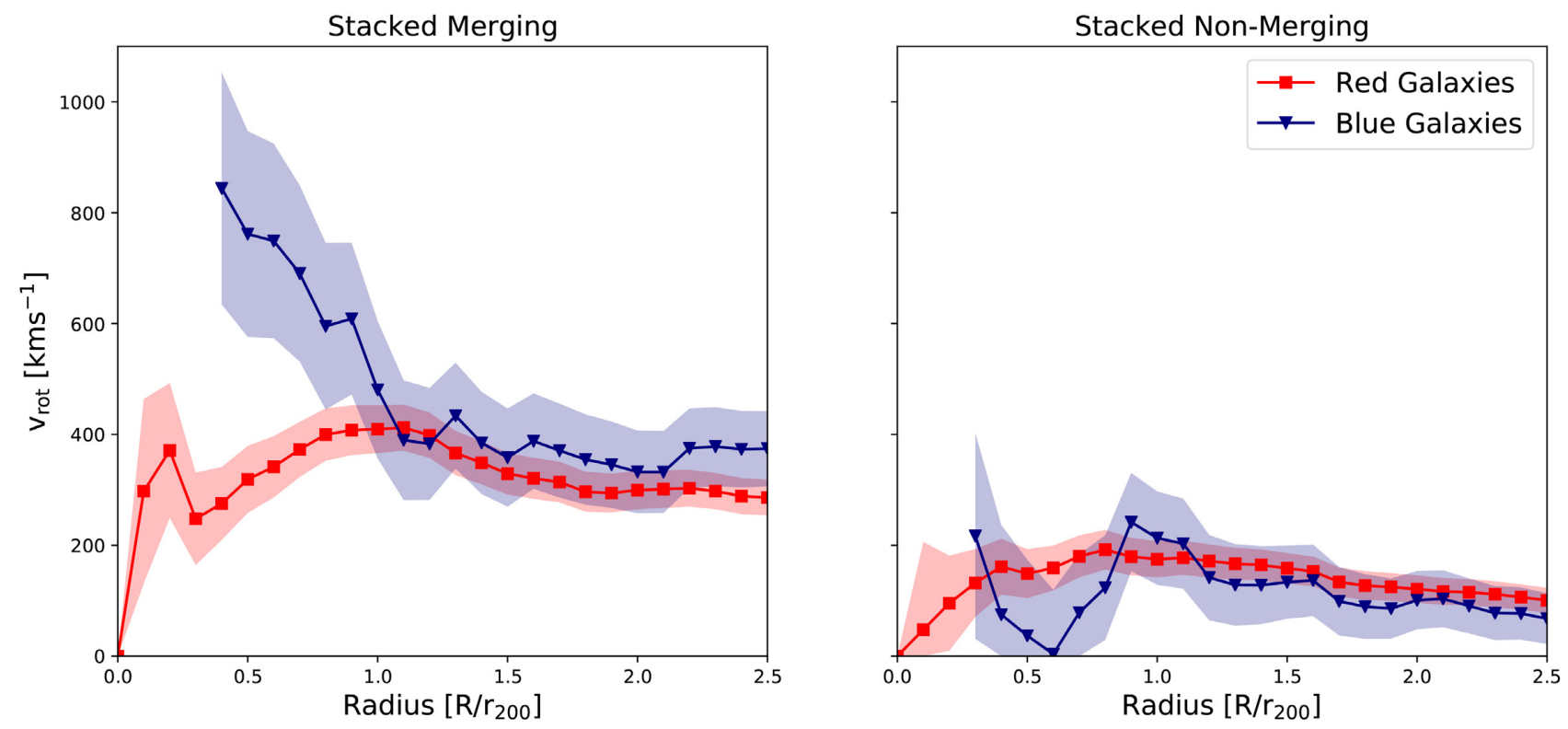

Figure 12. Constrained colour composite rotational profiles, similar to Fig. 9, with only BAX clusters lying within redshifts of $0.03 \leq z<0.09$ and possessing $\mathrm{X}$-ray luminosities in the range $<6 \times 10^{44} \mathrm{erg} \mathrm{s}^{-1}$. Note the differences in $v_{\mathrm{rot}}$ magnitude, especially the blue sub-population in the non-merging composite; loss of signal with a retained shape for values $\lesssim 2.0 r_{200}$. The shaded regions represent the uncertainties derived from the propagated standard error as denoted in equation (4).

a fraction of the relative velocities of the cores being interpreted as rotation. This manifests itself as high 'peculiar' rotation rates at lower radii.

Rows A and B of Fig. 14 bear resemblance to the rotation of the dumbbell BCG clusters shown in Figs 4 and 5, with their rotation rates being high at low radii, but with rapid reductions to minimum values around $0.5 r_{200}$ and $r_{200}$, then finally increasing again at larger radii. The simulation is also in agreement with the prediction for Abell 3391's merger phase drawn from the observations. Based upon observed properties such as angular separation of the cores, rotation profile, and the level of substructuring, it has been concluded that it is in 'post-initial merger phase' as mentioned above. The simulation supports this conclusion as it is only possible to create a rotation profile similar to that of Abell 3391 during second infall of the clusters (shortly before, during and shortly after second core passage). Beyond this the bulk linear motions of the cores, although detectable, are only a minor component in the rotation profile, as can be seen in Fig. 15. Thus comparing Abell 3391 to the simulation suggests that its BCGs are well into their second infall but have not reached second apocentre. This conclusion is in 


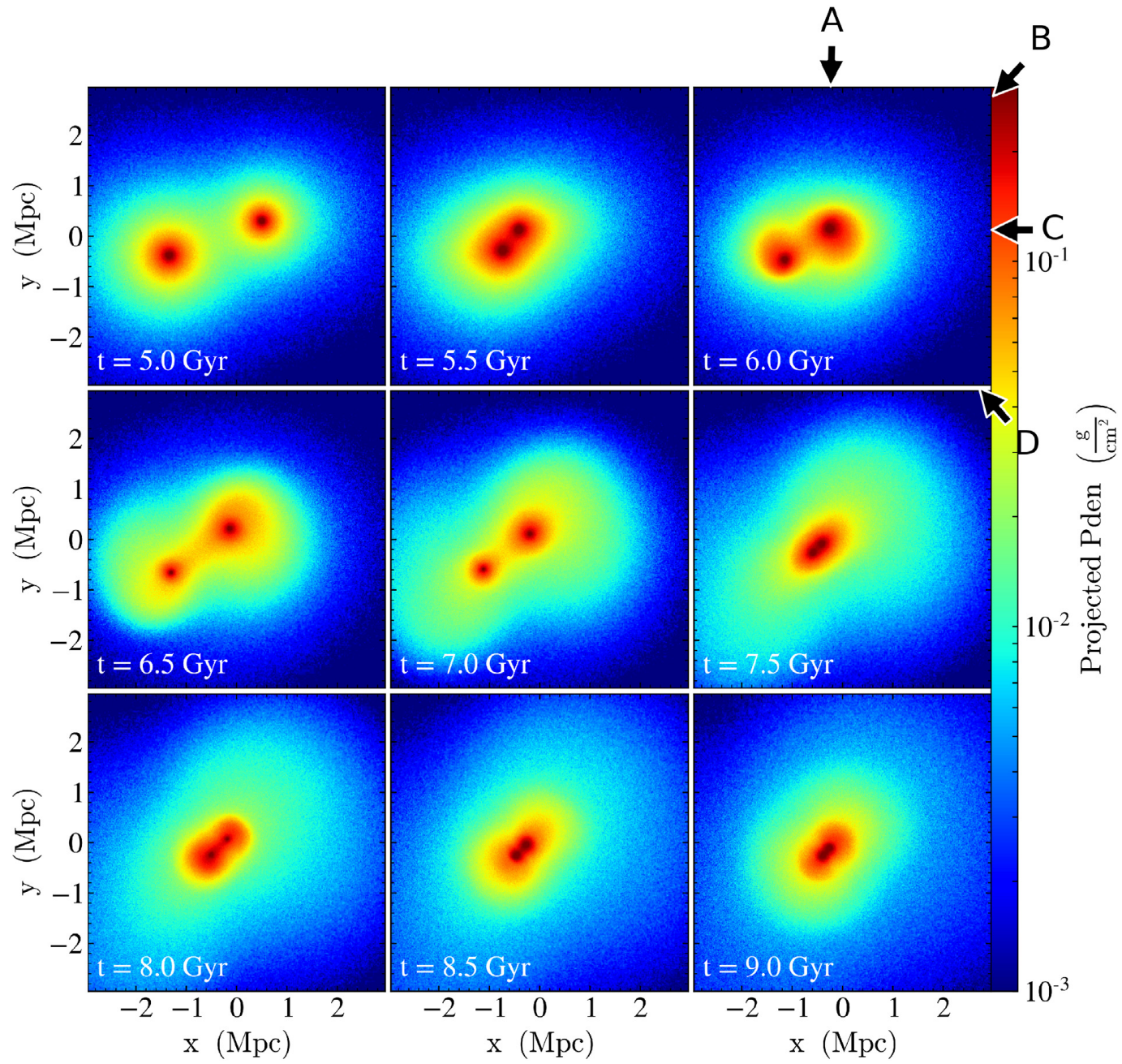

Figure 13. Evolution of the simulated cluster merger shown in projected particle density as a proxy for gravitational potential and line-of-sight galaxy distribution. The labelled arrows depict the four lines of sights from which the rotation of the system is measured throughout the merger. Line of sight A looks down the $y$-axis axis. B is down the line of second infall, $37 \mathrm{deg}$ from A. C is aliened with the $x$-axis. D is perpendicular to the line of second infall. All lines of sight are perpendicular to the global axis of rotation and are centred on maximum density.

agreement with that made from the observations, however it further constrains what stage the merger has progressed to.

Reverse engineering the previous section gives a framework that could assist observers to further identifying what merger phase a system is in and what angle the observations are being made from. Systems in which dumbbell BCGs are observed suggest an active mergers phase where the two potential wells can have made up to six passages through one another. A system that displays 'peculiar' central velocities along with dumbbell BCGs suggests very early phase mergers, in which the central potentials (BCGs) are on their second infall, i.e. shortly before or shortly after second passage. It also means the direction of observation is not perpendicular or parallel to the direction of motion of the dumbbell BCDs, neither is it parallel to the axis of rotation.

This simulation shows that if the viewing angle is favourable then the second infall of a major merger event, during which dumbbell BCGs would be observable, the linear re-infall of central galaxies creates dramatic changes to the observed radial rotation similar to those seen in the rotational profiles of the dumbbell BCG clusters in Figs 4 and 5. However it also shows that we should not expect such 'peculiar' rotation in all dumbbell BCG clusters. This is due, in part, to the phase that creates these rotations being relatively short lived
( $~ 500 \mathrm{Myr}$ ) when compared to the time period in which a dumbbell phase could be observed ( $\sim 2 \mathrm{Gyr})$. In addition to this, even if the observation was within the $500 \mathrm{Myr}$ window, any viewing angles aliened perpendicular or parallel to the linear motion of the re-infall are unable to detect the 'peculiar' rotation.

\section{DISCUSSION AND SUMMARY}

Despite the obvious caveat in the disparity between our merging and non-merging cluster sample sizes in this work, they still help to provide a consistency in our current understanding on the formation and evolution of galaxies within different cluster environments found in previous works. For example, the observed 'mixing' of the red and blue sub-populations of galaxies we see in Fig. 9 corresponds to rising velocity dispersion profiles of mixed red and blue ellipticals found in Bilton \& Pimbblet (2018); Mixing of sub-populations kinematically suggests substructured pre-processed galaxies are on infall as a result of galaxy-galaxy interactions (de Carvalho et al. 2017) either prior, or during, offaxial mergers between two sub-clusters; the pronounced population of late-type galaxies on infall in merging environments as inferred 

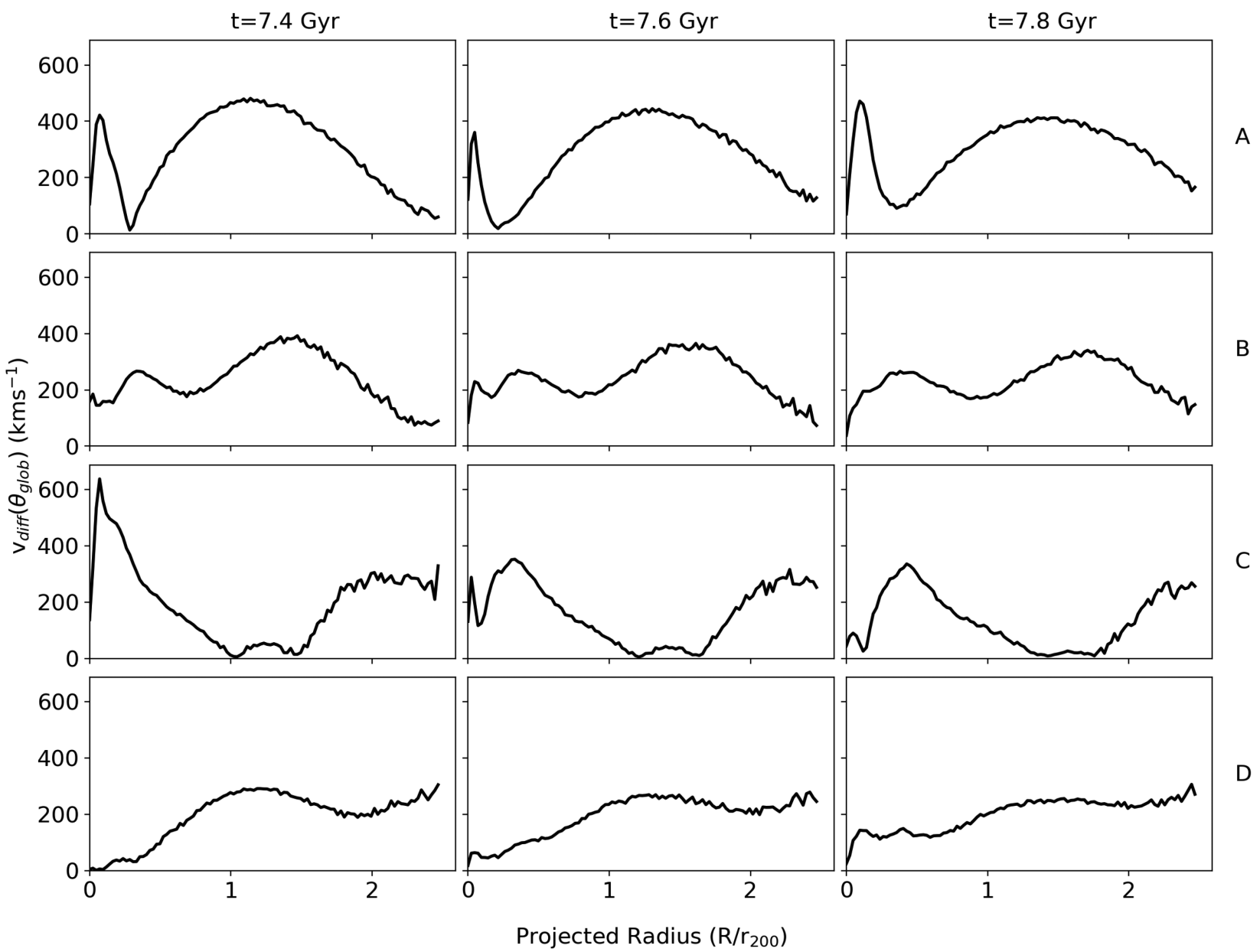

Figure 14. Rotational profiles throughout second core passage (7.4-7.8 Gyr) from different viewing angles. Radius is normalized to that of the $r_{200}$ of the more massive cluster. The top row shows the evolution for the line of sight down A (as depicted in Fig. 13) and the second, third, and fourth rows are down B, $\mathrm{C}$, and D, respectively. B and D which are parallel and perpendicular to the line of second infall display relatively continuous profiles whereas A and C show significant variation particularly within the core.

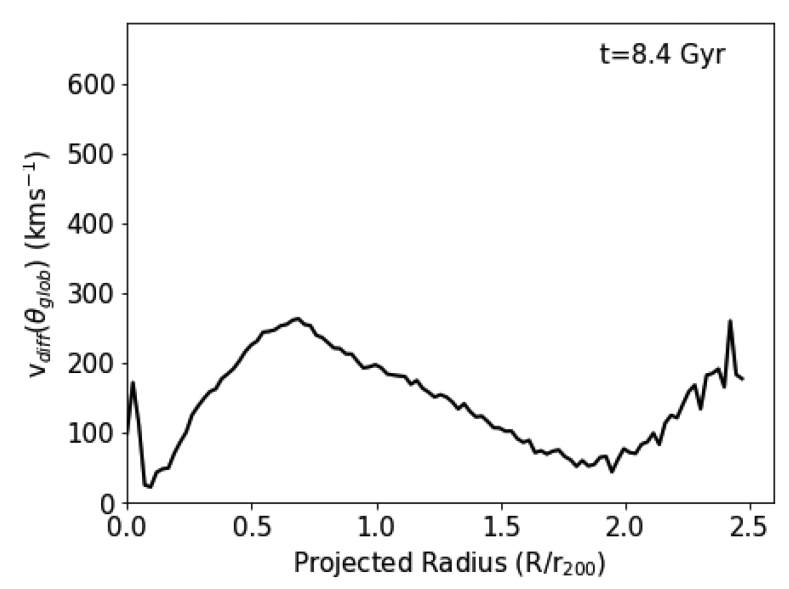

Figure 15. Rotational profiles for cluster merger during third core passage from line of sight $\mathrm{C}$ (as depicted in Fig. 13). The effect of the linear infall on the rotation of the cluster has become negligible, contributing only a $\sim 170 \mathrm{~km} \mathrm{~s}^{-1}$ increase in within the very core of the system.

by the blue sub-populations of galaxies gaining angular momentum $\lesssim r_{200}$, chiefly thought to be the result of galaxies with spiral morphology that have survived pre-processing (e.g. Cava et al. 2017; Bilton \& Pimbblet 2018; Costa, Ribeiro \& de Carvalho 2018;
Nascimento et al. 2019). The study of rotational profiles would have been aided by the addition of understanding how different morphological sub-populations of cluster galaxies contributed to each of the colour profiles. However, due to the limitations on resolving such features for every DR8 galaxy, no meaningful analysis could be conducted via the methodology we use within this work.

A common problem with observational studies of galaxy clusters is the limitation of the apparent 2D plane of sky and trying to ascertain information projected on to that sky. This inherently leads to projection effects due to our inability as observers to comprehend the precise angular and radial separations therefore determining the true direction of the rotational axis is not trivial. The main problem is trying to isolate the true mechanisms behind the observations we record in this work utilizing MP17's methods. All current observational techniques (e.g. Kalinkov et al. 2005; Hwang \& Lee 2007; Manolopoulou \& Plionis 2017) all determine a velocity gradient between some sort of observer defined axis. This observer defined axis within itself can be flawed as a result of our chosen centres, with this caveat in mind, we try and maintain consistency through using literary X-ray centres since they are commonly parallel to a cluster's potential. Therefore, this could potentially indicate that the various techniques we currently have at our disposal are specious, especially when considering we are trying to infer a variety of peculiar motions in a singular $z$ space. These are the same 
issues faced with our delineation between merging and non-merging environments via the Dressler \& Shectman (1988) $\Delta$-Test, where substructure is determined through local $z$-space deviations; does the presence of substructure genuinely infer rotation via angular momentum donation, or is this a mere deceptive emulation due to overlapping substructures biased by our limited ability to observe galaxy motion? Ideally, studies on global cluster rotation should combine and model observations between the ICM and the member galaxies; the collisional ICM leaves behind stronger markers of interaction and rotation than the more random (and therefore noisy) collisionless galaxies, which both operate on different time-scales (Roettiger \& Flores 2000). Furthermore, studies using the kinetic SZ-effect to simulate and analyse the motions of the ICM have shown that the angular momentum and direction between the ICM and DM both correlate significantly to imply DM dominance (Baldi et al. 2017, 2018).

Overlapping substructures in our projected space at least yields results for interactions of galaxies within the cluster. However, there is still the possibility of interloping substructures from other neighbouring clusters. This is in spite of the use of caustic techniques (Diaferio \& Geller 1997; Diaferio 1999) to estimate the mass profiles and membership, as well as removing heavily interloping substructures using the Einasto et al. (2001) catalogue. Some examples of interloping can be found in clusters such as Abell 2061 with possible infalling galaxies via a filament from Abell 2067 (Farnsworth et al. 2013); Abell 2065 is believed to be currently undergoing a merger with evidence of two independent subclusters with clear structure due to an unequal core merging event (Chatzikos, Sarazin \& Kempner 2006); Abell 3391 is in relatively close proximity to Abell 3395, with X-ray observations indicating the presence of a filament between the two clusters, highlighting the possibility of potential foreign foreground structures (Sugawara et al. 2017). Although, the use of the cluster caustics performs reasonably in delineating between cluster and non-cluster members, which offsets the reality of a few stragglers invading our cluster membership. The antithesis to this problem is that by applying our caustics to the more chaotic merging clusters from our sample we result in eliminating genuine cluster members due to the cluster galaxies gaining kinetic energy and increasing their interactions.

In this work we have acquired MPA-JHU DR8 galaxies crossmatched with a sample of galaxy clusters as defined by the BAX cluster data base to build their membership, which are stacked in accordance with their environments, as determined by the $\Delta$-test for substructure (Dressler \& Shectman 1988). This is complemented by NED galaxies of dumbbell clusters (Gregorini et al. 1992, 1994) to allow for comparisons of the dynamics from more extreme and complex systems. Finally, we compare our perspective rotation methodology from MP17 between our observational DR8 and NED data against FLASH 3D hydrodynamic and $N$-body simulations of merging clusters (Fryxell et al. 2000).

The key results are summarized as follows:

(i) Cluster rotation $v_{\text {rot }}$ profiles show consistently high rotation until $\sim r_{200}$ with the merging cluster environments (relaxing clusters), whereas non-merging environments commonly depict low $v_{\text {rot }}$ profiles indicative of relaxed clusters undergoing a reduction in the sloshing of galaxies caused by dynamical friction.

(ii) Merging cluster environments in our stack exhibit strong core rotation $\left(\lesssim 0.5 r_{200}\right)$ by the red galaxy sub-population, inferring a sloshing of evolved galaxies as they relax on to a common potential.

(iii) The blue galaxy sub-populations in our merging cluster stack have a high $v_{\text {glob }}$ segregation from the red galaxy sub-population in the core regions $\left(0.4 \lesssim R / r_{200} \lesssim 1.0 r_{200}\right)$ before homogenizing with the red sub-population, this may be a consequence of pre-processed subgroupings that are on infall.

(iv) The presence of multicore dumbbell BCGs in clusters displaying variable $v_{\text {glob }}$ profiles as a result of large peculiar velocities, in situ of the cluster's rest frame, is indicative of a recent core merger between two originally independent sub-clusters.

(v) Peculiar rotation velocities in dumbbell BCGs are a result of second infall of core galaxies along a linear trajectory that is not aligned with or perpendicular to the line of sight.

(vi) The presence of the peculiar rotation velocities are not obligatory in dumbbell BCG clusters due to phases of the dumbbells existence that do not have significant effects on the profile. in addition there are viewing angles that are incapable of measuring the linear motion as the peculiar rotation.

\section{ACKNOWLEDGEMENTS}

We would like to extend our thanks to the anonymous referee for their comments and suggestions during peer review of this work.

KAP and ER acknowledge the support of the Science and Technology Facilities Council (STFC) through the University of Hull's Consolidated Grant ST/R000840/1.

This research made use of Astropy, a community-developed core PYTHON package for Astronomy (Astropy Collaboration 2013, 2018).

This research has made use of the X-rays Clusters Database (BAX) which is operated by the Laboratoire d'Astrophysique de Tarbes-Toulouse (LATT), under contract with the Centre National d'Etudes Spatiales (CNES).

This research has made use of the NASA/IPAC Extragalactic Database (NED), which is operated by the Jet Propulsion Laboratory, California Institute of Technology, under contract with the National Aeronautics and Space Administration.

This research has made use of the 'K-corrections calculator' service available at http://kcor.sai.msu.ru/.

Funding for SDSS-III has been provided by the Alfred P. Sloan Foundation, the Participating Institutions, the National Science Foundation, and the U.S. Department of Energy Office of Science. The SDSS-III web site is http://www.sdss3.org/. SDSS-III is managed by the Astrophysical Research Consortium for the Participating Institutions of the SDSS-III Collaboration including the University of Arizona, the Brazilian Participation Group, Brookhaven National Laboratory, Carnegie Mellon University, University of Florida, the French Participation Group, the German Participation Group, Harvard University, the Instituto de Astrofisica de Canarias, the Michigan State/Notre Dame/JINA Participation Group, Johns Hopkins University, Lawrence Berkeley National Laboratory, Max Planck Institute for Astrophysics, Max Planck Institute for Extraterrestrial Physics, New Mexico State University, New York University, Ohio State University, Pennsylvania State University, University of Portsmouth, Princeton University, the Spanish Participation Group, University of Tokyo, University of Utah, Vanderbilt University, University of Virginia, University of Washington, and Yale University.

\section{REFERENCES}

Aihara H. et al., 2011, ApJS, 193, 29

Astropy Collaboration, 2013, A\&A, 558, A33

Astropy Collaboration, 2018, AJ, 156, 123 
Bahé Y. M., McCarthy I. G., Balogh M. L., Font A. S., 2013, MNRAS, 430, 3017

Baldi A. S., De Petris M., Sembolini F., Yepes G., Lamagna L., Rasia E., 2017, MNRAS, 465, 2584

Baldi A. S., De Petris M., Sembolini F., Yepes G., Cui W., Lamagna L., 2018, MNRAS, 479, 4028

Bamford S. P. et al., 2009, MNRAS, 393, 1324

Beers T. C., Flynn K., Gebhardt K., 1990, AJ, 100, 32

Berrier J. C., Stewart K. R., Bullock J. S., Purcell C. W., Barton E. J., Wechsler R. H., 2009, ApJ, 690, 1292

Bilton L. E., Pimbblet K. A., 2018, MNRAS, 481, 1507

Böhringer H. et al., 2000, ApJS, 129, 435

Bond J. R., Kofman L., Pogosyan D., 1996, Nature, 380, 603

Brinchmann J., Charlot S., White S. D. M., Tremonti C., Kauffmann G., Heckman T., Brinkmann J., 2004, MNRAS, 351, 1151

Caglar T., Hudaverdi M., 2017, MNRAS, 472, 2633

Cava A. et al., 2017, A\&A, 606, A108

Chatzikos M., Sarazin C. L., Kempner J. C., 2006, ApJ, 643, 751

Collins C. A. et al., 2009, Nature, 458, 603

Costa A. P., Ribeiro A. L. B., de Carvalho R. R., 2018, MNRAS, 473, L31

David L. P., Forman W., Jones C., 1999, ApJ, 519, 533

de Carvalho R. R., Ribeiro A. L. B., Stalder D. H., Rosa R. R., Costa A. P., Moura T. C., 2017, AJ, 154, 96

Diaferio A., 1999, MNRAS, 309, 610

Diaferio A., Geller M. J., 1997, ApJ, 481, 633

Dressler A., 1980, ApJ, 236, 351

Dressler A., Shectman S. A., 1988, AJ, 95, 985

Dubinski J., 1998, ApJ, 502, 141

Ebeling H., Edge A. C., Bohringer H., Allen S. W., Crawford C. S., Fabian A. C., Voges W., Huchra J. P., 1998, MNRAS, 301, 881

Einasto M., Einasto J., Tago E., Müller V., Andernach H., 2001, AJ, 122, 2222

Farnsworth D., Rudnick L., Brown S., Brunetti G., 2013, ApJ, 779, 189

Feast M. W., Thackeray A. D., Wesselink A. J., 1961, MNRAS, 122, 433

Fryxell B. et al., 2000, ApJS, 131, 273

Geller M. J., Beers T. C., 1982, PASP, 94, 421

Gifford D., Miller C. J., 2013, ApJ, 768, L32

Gifford D., Miller C., Kern N., 2013, ApJ, 773, 116

Godłowski W., Szydłowski M., Flin P., Biernacka M., 2003, Gen. Relativ. Gravit., 35, 907

Godłowski W., Szydłowski M., Flin P., 2005, Gen. Relativ. Gravit., 37, 615

Gómez P. L. et al., 2003, ApJ, 584, 210

Gregorini L., Vettolani G., de Ruiter H. R., Parma P., 1992, A\&AS, 95, 1

Gregorini L., de Ruiter H. R., Parma P., Sadler E. M., Vettolani G., Ekers R. D., 1994, A\&AS, 106, 1

Gunn J. E., Gott J. R., III, 1972, ApJ, 176, 1

Haines C. P. et al., 2015, ApJ, 806, 101

Hernquist L., 1990, ApJ, 356, 359

Hogg D. W. et al., 2003, ApJ, 585, L5

Hogg D. W. et al., 2004, ApJ, 601, L29

Hwang H. S., Lee M. G., 2007, ApJ, 662, 236

Jin S.-W., Gu Q., Huang S., Shi Y., Feng L.-L., 2014, ApJ, 787, 63

Jones C., Forman W., 1999, ApJ, 511, 65

Kalinkov M., Valchanov T., Valtchanov I., Kuneva I., Dissanska M., 2005, MNRAS, 359, 1491

Kauffmann G. et al., 2003, MNRAS, 341, 33

Kravtsov A. V., Borgani S., 2012, ARA\&A, 50, 353

Laine S., van der Marel R. P., Lauer T. R., Postman M., O’Dea C. P., Owen F. N., 2003, AJ, 125, 478
Lakhchaura K., Singh K. P., Saikia D. J., Hunstead R. W., 2013, ApJ, 767, 91

Lauer T. R., 1988, ApJ, 325, 49

Lewis I. et al., 2002, MNRAS, 334, 673

Li L.-X., 1998, Gen. Relativ. Gravit., 30, 497

Manolopoulou M., Plionis M., 2017, MNRAS, 465, 2616

Marini F. et al., 2004, MNRAS, 353, 1219

Materne J., Hopp U., 1983, A\&A, 124, L13

Merritt D., 1985, ApJ, 289, 18

Moore B., Lake G., Quinn T., Stadel J., 1999, MNRAS, 304, 465

Nascimento R. S., Lopes P. A. A., Ribeiro A. L. B., Costa A. P., Morell D. F., 2019, MNRAS, 483, L121

Oegerle W. R., Hill J. M., 1992, AJ, 104, 2078

Oemler A., Jr., 1974, ApJ, 194, 1

Ostriker J. P., Tremaine S. D., 1975, ApJ, 202, L113

Peebles P. J. E., 1969, ApJ, 155, 393

Pimbblet K. A., 2008, Publ. Astron. Soc. Aust., 25, 176

Pimbblet K. A., 2011, MNRAS, 411, 2637

Pimbblet K. A., Roseboom I. G., Doyle M. T., 2006, MNRAS, 368, 651

Pinkney J., Roettiger K., Burns J. O., Bird C. M., 1996, ApJS, 104, 1

Poggianti B. M. et al., 2017, ApJ, 844, 48

Poole G. B., Fardal M. A., Babul A., McCarthy I. G., Quinn T., Wadsley J., 2006, MNRAS, 373, 881

Popesso P., Biviano A., Böhringer H., Romaniello M., 2007, A\&A, 461, 397

Postman M., Geller M. J., 1984, ApJ, 281, 95

Quintana H., Lawrie D. G., 1982, AJ, 87, 1

Quintana H., Ramirez A., Way M. J., 1996, AJ, 112, 36

Regos E., Geller M. J., 1989, AJ, 98, 755

Reiprich T. H., Böhringer H., 2002, ApJ, 567, 716

Ricker P. M., 1998, ApJ, 496, 670

Roettiger K., Flores R., 2000, ApJ, 538, 92

Roettiger K., Loken C., Burns J. O., 1997, ApJS, 109, 307

Sadat R., Blanchard A., Kneib J.-P., Mathez G., Madore B., Mazzarella J. M., 2004, A\&A, 424, 1097

Shan H., Qin B., Fort B., Tao C., Wu X.-P., Zhao H., 2010, MNRAS, 406, 1134

Sheen Y.-K. et al., 2017, ApJ, 840, L7

Smith G. P., Kneib J.-P., Smail I., Mazzotta P., Ebeling H., Czoske O., 2005 , MNRAS, 359, 417

Song H., Hwang H. S., Park C., Smith R., Einasto M., 2018, ApJ, 869, 124

Springel V. et al., 2005, Nature, 435, 629

Sugawara Y., Takizawa M., Itahana M., Akamatsu H., Fujita Y., Ohashi T., Ishisaki Y., 2017, PASJ, 69, 93

Tormen G., 1997, MNRAS, 290, 411

Tovmassian H. M., 2015, Astrophysics, 58, 328

Tremonti C. A. et al., 2004, ApJ, 613, 898

Vitvitska M., Klypin A. A., Kravtsov A. V., Wechsler R. H., Primack J. R., Bullock J. S., 2002, ApJ, 581, 799

von der Linden A., Wild V., Kauffmann G., White S. D. M., Weinmann S., 2010, MNRAS, 404, 1231

White D. A., Fabian A. C., 1995, MNRAS, 273, 72

Wright E. L., 2006, PASP, 118, 1711

ZuHone J. A., 2011, ApJ, 728, 54

This paper has been typeset from a $\mathrm{TE}_{\mathrm{E}} / \mathrm{LT} \mathrm{E} \mathrm{X}$ file prepared by the author. 